\title{
Calibration of moving puncture simulations
}

\author{
Bernd Brügmann, ${ }^{1}$ José A. González, ${ }^{1,2}$ Mark Hannam, ${ }^{1}$ Sascha Husa, ${ }^{1}$ and Ulrich Sperhake ${ }^{1}$ \\ ${ }^{1}$ Theoretical Physics Institute, University of Jena, 07743 Jena, Germany \\ ${ }^{2}$ Instituto de Física y Matemáticas, Universidad Michoacana de San Nicolas de Hidalgo, Edificio C-3, \\ Cd. Universitaria. C.P. 58040 Morelia, Michoacán, México \\ Wolfgang Tichy \\ Department of Physics, Florida Atlantic University, Boca Raton, Florida 33431, USA
}

(Received 3 November 2006; published 16 January 2008)

\begin{abstract}
We present single and binary black-hole simulations that follow the "moving-puncture" paradigm of simulating black-hole spacetimes without excision, and use "moving boxes" mesh refinement. Focusing on binary black-hole configurations where the simulations cover roughly two orbits, we address five major issues determining the quality of our results: numerical discretization error, finite extraction radius of the radiation signal, physical appropriateness of initial data, gauge choice, and computational performance. We also compare results we have obtained with the BAM code described here with the independent LEAN code.
\end{abstract}

DOI: 10.1103/PhysRevD.77.024027

PACS numbers: 04.25.dg, 04.30.Db, 95.30.Sf

\section{INTRODUCTION}

More than 40 years after the first numerical simulations of binary black-hole dynamics $[1,2]$, the numerical relativity community is now ready to compare binary black-hole simulations with experimental data. A series of recent breakthroughs [3-7] has lead to a phase transition in the field: long-term evolutions of inspiraling black holes that last for one orbit and more have been obtained with several independent codes [6-13], and accurate gravitationalwave signals have been computed.

Coincidentally, these breakthroughs parallel the first science runs of large-scale interferometric gravitationalwave observatories at design sensitivity [14]. The inspiral and merger of black-hole binaries are considered to be among the most promising sources for this current generation of Earth-based gravitational-wave detectors, and it has become feasible for numerical relativity to contribute to the analysis of experimental data.

Such contributions will require large-scale parameter studies, and correspondingly large computational resources. The crucial current technical problem in the field is the efficiency of the simulations, and the establishment of a "data analysis pipeline," connecting analytical calculations of the early inspiral and late ring-down phases with numerical simulations, and especially with gravitationalwave searches in actual detector data.

In this paper we present a new version of the BAM code $[6,15,16]$ for binary black-hole simulations that follows the "moving-puncture" [4,5] paradigm of simulating blackhole spacetimes without excision, and use "moving boxes" mesh refinement. We give a detailed presentation of our methods, which will serve as a reference for future work, and we use simple test cases of single and orbiting black holes to calibrate our methods.
We give a detailed discussion of convergence and accuracy of our code, and address further issues determining the quality of our results: finite extraction radius of the radiation signal, physical appropriateness of initial-data parameters, gauge choice, and computational performance. We compare evolutions from different initial-data parameters and conclude that post-Newtonian (PN) methods provide excellent estimates for initial positions, momenta, and masses for quasicircular orbits in the nonspinning equalmass case, removing the necessity of complex initial-data studies. We present new results concerning the damping parameter $\eta$ in the popular $\tilde{\Gamma}$-driver shift, which was originally introduced to handle long-term coordinate drifts, but is now found to also have the beneficial effect of magnifying the size of the apparent horizons and thus changing the effective spatial resolution near the black hole, and additionally reduces noise in the puncture motion and waveforms. We also present timing and performance results: we have been able to perform some of our highest resolution runs at a computational cost of $\approx 500 \mathrm{CPU}$ hours (see Table II), giving rise to the hope that numerical relativity will indeed be capable of large-scale parameter studies.

In Sec. II we recall the basic equations of the movingpuncture method, followed by a detailed description of our wave-extraction algorithm and computation of ArnowittDeser-Misner (ADM) and Bondi quantities in Sec. III. Our numerical methods and code structure are presented in Sec. IV. In Secs. V, VI, and VII we describe our results for single black holes, orbiting black holes evolved using standard quasicircular-orbit initial-data parameters (allowing direct comparison with the LEAN code [13]), and orbiting black holes with alternative initial-data parameters. We conclude with a discussion in Sec. VIII. 


\section{PUNCTURE METHOD AND MOVING PUNCTURES}

\section{A. Initial data}

We will model $N$ black-hole initial data by adopting the Brill-Lindquist wormhole topology [18] with $N+1$ asymptotically flat ends for our initial geometry, thus enforcing the presence of $N$ "throats." The asymptotically flat ends are compactified and identified with points $r_{i}$ on $R^{3}$. The coordinate singularities at the points $r_{i}$ resulting from compactification are referred to as punctures. In the context of initial data, punctures have been extensively studied and the treatment of the singularity in the constraint equations is well understood [19]. From a physical point of view the puncture representation of black-hole initial data is particularly appealing because it provides a simple prescription for associating masses, momenta, and spins with any number of black holes.

Initial data consist of the positive-definite metric and extrinsic curvature $\left(g_{i j}, K_{i j}\right)$ induced on a spatial hypersurface $\Sigma$ with timelike unit normal $n^{i}$. We choose our sign conventions as $n^{i} n_{i}=-1$,

$$
K_{a b}=-\frac{1}{2 \alpha} \mathcal{L}_{n} g_{a b} .
$$

We construct these data using the conformal transversetraceless decomposition of the initial-value equations, outlined in [20], and related to other conformal decompositions in [21]. The spatial metric and intrinsic curvature are conformally related to counterparts on a background space via an initial conformal factor $\psi_{0}$, and the conformal extrinsic curvature is split into trace and trace-free parts:

$$
\begin{gathered}
g_{i j}=\psi_{0}^{4} \tilde{g}_{i j}, \\
K_{i j}=\psi_{0}^{-2} \bar{A}_{i j}+\frac{1}{3} g_{i j} K,
\end{gathered}
$$

where $K=g^{i j} K_{i j}$ and $\bar{A}_{i j}$ is trace free.

We choose an initially flat background metric, $\tilde{g}_{i j}=\delta_{i j}$, and a maximal slice, $K=0$. The second choice decouples the Hamiltonian and momentum constraints. The momentum constraint now takes the form

$$
\partial_{j} \bar{A}_{i j}=0
$$

(where repeated indices are summed), and admits the Bowen-York solutions [22] for any number of black holes with prescribed ADM linear and angular momenta.

The Hamiltonian constraint becomes an elliptic equation for the conformal factor with a solution of the form $[19,23-$ 27]

$$
\begin{gathered}
\psi_{0}=\psi_{B L}+u, \\
\psi_{B L}=1+\sum_{i=1}^{N} \frac{m_{i}}{2 r_{i}} .
\end{gathered}
$$

The function $u$ is determined by an elliptic equation on $R^{3}$ and is $C^{\infty}$ everywhere except at the punctures, where it is $C^{2}$. The $m_{i}$ parametrize the mass of each black hole, but actually equal the total mass of the black hole only in the special case of the Schwarzschild spacetime. In the general case, the ADM mass at the $i$ th asymptotically flat end (i.e., the puncture) is given by

$$
M_{i}=m_{i}\left(1+u_{i}+\sum_{i \neq j} \frac{m_{j}}{2 d_{i j}}\right)
$$

where $u_{i}$ is the value of $u$ at the $i$ th puncture, and $d_{i j}$ is the coordinate distance between punctures $i$ and $j$. The ADM puncture mass can in general differ from the apparent horizon mass. In actual examples, however, it has been found to agree within numerical uncertainty with the mass $M_{A H, i}$ calculated from the area of the apparent horizon, $A$, by

$$
M_{A H, i}=\sqrt{\frac{A}{16 \pi}}
$$

for nonspinning punctures [28], and for spinning punctures we have found it to agree numerically with the black-hole mass given by a modification of the Christodoulou formula [29],

$$
M_{i}^{2}=\left(M_{A H, i}\right)^{2}+\frac{S_{i}^{2}}{4\left(M_{A H, i}\right)^{2}} .
$$

Throughout this paper, lowercase $m_{i}$ will refer to the mass parameter in the ansatz (5), and $M_{i}$ will refer to the blackhole mass given by Eq. (6). When we desire particular values of $M_{i}$, we make initial guesses of $m_{i}$ by inverting Eq. (6), and iteratively improve the $m_{i}$ based on successive values of $u_{i}$ until the $M_{i}$ equal the desired values to within $0.02 \%$. We will denote by $M=\sum_{i} M_{i}$ the total black-hole mass of the system under investigation, and typically use $M$ as the mass scale for quoting results (e.g., to express time or distance in terms of a mass scale).

To complete the definition of the initial data, we also need to specify initial values for our gauge quantities, the lapse function $\alpha$, and shift vector $\beta^{i}$. At time $t=0$ we use

$$
\begin{gathered}
\alpha=1 \quad \text { or } \quad \alpha=\psi_{0}^{-2}, \\
\beta^{i}=0 .
\end{gathered}
$$

Both choices for the lapse have been used successfully, although the "precollapsed" lapse suggested in [4,30] is found to reduce initial gauge dynamics and is our standard choice. Both lapse and shift are updated by evolution equations depending on the physical variables, as described below. 


\section{B. Evolution system}

We evolve the initial data with the Baumgarte-ShapiroShibata-Nakamura (BSSN) system [31,32]. On the initial slice the standard BSSN variables $\phi, \tilde{g}_{i j}, \tilde{A}_{i j}, K$, and $\tilde{\Gamma}^{i}$ are related to the variables in the conformal transversetraceless decomposition by

$$
\begin{gathered}
\phi=\ln \psi_{0} \\
\tilde{A}_{i j}=\psi^{-6} \bar{A}_{i j} \\
\tilde{\Gamma}^{i}=-\partial_{j} \tilde{g}^{i j},
\end{gathered}
$$

and $\tilde{g}_{i j}$ and $K$ are unchanged. These variables are evolved using

$$
\begin{gathered}
\partial_{0} \phi=-\frac{1}{6} \alpha K, \\
\partial_{0} \tilde{g}_{i j}=-2 \alpha \tilde{A}_{i j}, \\
\partial_{0} \tilde{A}_{i j}=e^{-4 \phi}\left[-D_{i} D_{j} \alpha+\alpha R_{i j}\right]^{T F}+\alpha\left(K \tilde{A}_{i j}-2 \tilde{A}_{i k} \tilde{A}_{j}^{k}\right), \\
\partial_{0} K=-D^{i} D_{i} \alpha+\alpha\left(\tilde{A}_{i j} \tilde{A}^{i j}+\frac{1}{3} K^{2}\right), \\
+\frac{2}{3} \tilde{\Gamma}^{i}=\tilde{g}^{j k} \partial_{j} \partial_{k} \beta^{i}+\frac{1}{3} \tilde{g}^{i j} \partial_{j} \partial_{k} \beta^{k}+2 \tilde{A}^{i j} \partial_{j} \alpha+2 \alpha\left(\tilde{\Gamma}_{j} \tilde{\Gamma}^{i}-\tilde{\Gamma}^{j} \tilde{A}_{j} \tilde{\beta}^{i k}+6 \tilde{A}^{i j} \partial_{j} \phi\right. \\
\left.-\frac{2}{3} \tilde{g}^{i j} \partial_{j} K\right),
\end{gathered}
$$

where $\partial_{0}=\partial_{t}-\mathcal{L}_{\beta}, D_{i}$ is the covariant derivative with respect to the conformal metric $\tilde{g}_{i j}$, and " $T F$ " denotes the trace-free part of the expression with respect to the physical metric, $X_{i j}^{T F}=X_{i j}-\frac{1}{3} g_{i j} X_{k}^{k}$. The Ricci tensor $R_{i j}$ is given by

$$
\begin{gathered}
R_{i j}=\tilde{R}_{i j}+R_{i j}^{\phi}, \\
\tilde{R}_{i j}=-\frac{1}{2} \tilde{g}^{l m} \partial_{l} \partial_{m} \tilde{g}_{i j}+\tilde{g}_{k(i} \partial_{j} \tilde{\Gamma}^{k}+\tilde{\Gamma}^{k} \tilde{\Gamma}_{(i j) k} \\
+\tilde{g}^{l m}\left(2 \tilde{\Gamma}_{l(i}^{k} \tilde{\Gamma}_{j) j m}+\tilde{\Gamma}_{i m}^{k} \tilde{\Gamma}_{k l j}\right), \\
R_{i j}^{\phi}=-2 D_{i} D_{j} \phi-2 \tilde{g}_{i j} D^{k} D_{k} \phi+4 D_{i} \phi D_{j} \phi \\
-4 \tilde{g}_{i j} D^{k} \phi D_{k} \phi .
\end{gathered}
$$

Note that $\tilde{g}_{i j}$ and $\tilde{A}_{i j}$ are tensor densities of weight $-2 / 3$ and $\phi$ is the logarithm of a tensor density of weight $1 / 6$, so their Lie derivatives are

$$
\begin{aligned}
& \mathcal{L}_{\beta} \phi=\beta^{k} \partial_{k} \phi+\frac{1}{6} \partial_{k} \beta^{k}, \\
& \mathcal{L}_{\beta} \tilde{g}_{i j}=\beta^{k} \partial_{k} \tilde{g}_{i j}+\tilde{g}_{i k} \partial_{j} \beta^{k}+\tilde{g}_{j k} \partial_{i} \beta^{k}-\frac{2}{3} \tilde{g}_{i j} \partial_{k} \beta^{k}, \\
& \mathcal{L}_{\beta} \tilde{A}_{i j}=\beta^{k} \partial_{k} \tilde{A}_{i j}+\tilde{A}_{i k} \partial_{j} \beta^{k}+\tilde{A}_{j k} \partial_{i} \beta^{k}-\frac{2}{3} \tilde{A}_{i j} \partial_{k} \beta^{k} .
\end{aligned}
$$

It is common to evolve the BSSN system as a partially constrained scheme, where one or both of the algebraic constraints $\operatorname{det}(\tilde{g})=1$ and $\operatorname{Tr}\left(\tilde{A}_{i j}\right)=0$ are enforced at every full or intermediate time step of the evolution scheme. This has been found to be necessary to obtain stable, accurate evolutions of black-hole punctures in several cases; see, e.g., [30,33]. Likewise, the algebraic constraints and the first-order differential constraint $\tilde{\Gamma}^{i}=-\partial_{j} \tilde{g}^{i j}$ can be used for the source terms without affecting well-posedness, but changing, for example, the source terms of the constraint-propagation equations. In the BAM code we make the following choices:

(i) Wherever $\tilde{\Gamma}^{i}$ appears undifferentiated, we explicitly use $\tilde{\Gamma}^{i}=-\partial_{j} \tilde{g}^{i j}$ instead of the evolved variable $\tilde{\Gamma}^{i}$. Otherwise, $\tilde{\Gamma}^{i}$ is used.

(ii) The algebraic constraints $\operatorname{Tr}\left(\tilde{A}_{i j}\right)=0$ and $\operatorname{det}\left(\tilde{g}_{i j}\right)=$ 1 are imposed whenever the right-hand sides are calculated, and also at the end of each evolution step. (Imposing the algebraic constraints at each evolution ministep does not imply that they will hold after each full time step, because of the nonlinearity of the expressions involved.)

Further important choices concern the treatment of the conformal factor and the gauge choice. We will describe below the most popular choices that have been used successfully in the literature, and present some comparisons of different choices in Secs. V and VI.

\section{Choices for the conformal factor}

Let us first recall the fixed-puncture method, where the puncture pole is treated analytically and the punctures do not move. This is described in detail in [30]. The BSSN conformal factor is split according to

$$
\phi=\ln \psi=\xi+\ln \psi_{B L},
$$

where $\xi$ is assumed to be regular at the puncture. In an evolution the regular function $\xi$ is evolved via the corresponding version of Eq. (14), and the logarithmically singular part $\ln \psi_{B L}$ is kept constant. The key issue in the whole approach is to show that all evolved variables remain sufficiently regular at the punctures during evolution. In [30], a detailed analysis is given in terms of power counting arguments.

The disadvantage of this method is that it assumes a natural split of the conformal factor according to Eq. (22) throughout an evolution, i.e., that the slices remain connected to all asymptotically flat ends.

In the moving-puncture approach the entire conformal factor is evolved. No assumption is made about the geometry of the slices. The slices are now allowed to approach whatever geometry is preferred by the gauge conditions. It turns out that in this preferred geometry the conformal factor does not maintain the $1 / r$ Brill-Lindquist pole, and instead develops a $1 / \sqrt{r}$ pole near the "punc- 
ture" [34]. The puncture ceases to represent a second infinity, and instead corresponds to a surface inside the horizon. The space outside this surface can be sufficiently accurately resolved with a finite-difference code. We can then regard the moving-puncture method not as a mere trick to prolong the lifetime of a black-hole evolution, but rather as an elegant and simple alternative to excision techniques: the singularity is not cut out of the numerical grid, it is avoided by the choice of gauge [34].

The question now is how to evolve the divergent conformal factor. In practice two proposals have been found to work, which we will call the $\phi$ method and the $\chi$ method. In the $\phi$ method [5], one works directly with the original $\mathrm{BSSN}$ variable $\phi$,

$$
\phi=\ln \psi,
$$

and the evolution system remains as Eqs. (14)-(18). The purely experimental result is that finite differencing across the $\ln (r)$ singularity at $r=0$ leads to stable evolutions.

In the $\chi$ method [4], a new conformal factor is defined that is finite at the puncture,

$$
\begin{gathered}
\chi=\psi^{-4}, \\
\partial_{0} \chi=\frac{2}{3} \chi \alpha K, \quad \mathcal{L}_{\beta} \chi=\beta^{k} \partial_{k} \chi-\frac{2}{3} \chi \partial_{k} \beta^{k} .
\end{gathered}
$$

Now Eq. (25) replaces Eq. (14) in the evolution system. If $\psi$ has the usual $1 / r$ pole at the puncture, then $\chi=O\left(r^{4}\right)$ at the puncture. As discussed in [34], the behavior changes to $\chi=O\left(r^{2}\right)$ during the evolution.

This approach does not rely on finite differencing of a singularity, but the singular structure of the black hole is incorporated in the vanishing of $\chi$ at the puncture. Because of divisions by $\chi$ in the evolution equations, care needs to be taken in the numerical implementation to avoid divisions by zero or discontinuities arising from unphysically negative values of $\chi$. We find that these problems can be avoided if $\chi$ is consistently replaced in the right-hand sides of (14)-(18) by $\max (\chi, \epsilon)$ (for some small $\epsilon$ ) wherever divisions by $\chi$ occur. As a general rule, we choose $\epsilon$ as follows. Near the puncture, we know that $\chi \sim(2 r / m)^{4}$ in the initial data, and later $\chi$ evolves to the form $\chi \sim$ $(2 r / m)^{2}$. We therefore expect that $\chi$ will not fall far below its initial minimum value, and choose $\epsilon$ to be less than $\left(2 r_{\min } / m\right)^{4}$.

\section{Choices for the gauge}

The second ingredient in the moving-puncture method is a modification to the gauge choice. Both approaches now rely on the "covariant" form of " $1+\log$ " slicing $[4,35]$,

$$
\left(\partial_{t}-\beta^{i} \partial_{i}\right) \alpha=-2 \alpha K .
$$

The shift advection term had been dropped in the version of " $1+\log$ " slicing used in the analytic fixed-puncture approach:

$$
\partial_{t} \alpha=-2 \alpha K \text {, }
$$

and also in one of the first versions of the moving-puncture approach of [5]. An attractive feature of Eq. (27) is that the slicing is asymptotically maximal for a stationary solution, such as the final Kerr black hole of a merged binary. However, Eq. (27) admits undesirable zero-speed modes in the BSSN system [36,37] and Eq. (26) turns out to be a better choice for moving-puncture evolutions $[4,11]$. The stationary Schwarzschild slicing with Eq. (26) is given in [34,38].

For the shift, we use a gamma-freezing condition. The original gamma-freezing condition introduced in [30] is

$$
\partial_{t} \beta^{i}=\frac{3}{4} B^{i}, \quad \partial_{t} B^{i}=\partial_{t} \tilde{\Gamma}^{i}-\eta B^{i} .
$$

Variants of this condition $[4,5,11,36,37]$ consist of replacing some or all of the $\partial_{t}$ derivatives with $\partial_{0}=\partial_{t}-\beta^{i} \partial_{i}$. We will label these options with reference to each of the three time derivatives in (28): " $t t t$ " denotes that $\partial_{t}$ is used for all three derivatives, " $0 t t$ " denotes that $\partial_{0}$ is used for the first time derivative, and $\partial_{t}$ for the other two, and so on. The properties of the different choices are studied in [36,37]. Reference [37] proves that the combination of the BSSN equations with the " $1+\log$ " slicing condition (26) and the 000 shift choice is strongly hyperbolic in the sense of first order in time, second order in space systems [39-43], and thus yields a well-posed initial-value problem. For the final results presented in Sec. VI we quote results obtained with the " $t t t$ " and " 000 " options, which are both found to yield stable evolutions. All our recent work is based on the manifestly hyperbolic choice 000 , i.e., we make the replacement $\partial_{t} \rightarrow \partial_{0}$ everywhere.

\section{ASYMPTOTICS}

\section{A. Using $\Psi_{\mathbf{4}}$ for wave extraction}

Extracting physical information from numerical simulations in general relativity represents a highly nontrivial task for two reasons. First, most of the functions numerically computed in the course of an evolution are inherently coordinate dependent. Second, quantities commonly used for the description of local systems in other areas of physics, such as energy and angular momentum, are hard to define in an unambiguous way in corresponding scenarios in general relativity. For the problem at hand, the most important physical information to be extracted is the energy and momenta radiated away in the form of gravitational waves and the precise shape of these gravitational waves as seen by a detector at large distances from the source.

In the past, the extraction of these quantities from numerical simulations has been performed using either the Zerilli-Moncrief (see, e.g., [44]) or the Newman-Penrose approach. In this work we focus on the calculation of the Newman-Penrose scalar $\Psi_{4}$. This method has been discussed frequently in the literature, but we provide a de- 
tailed description to make clear the conventions we use (which can lead, for example, to differences in signs or constant factors of two), and to provide a complete, selfcontained account of all the steps involved in calculating waveforms as well as radiated momenta and energy from the numerically evolved variables. For this purpose we will assume as known on a given hypersurface $t=$ const the ADM variables $g_{i j}$ and $K_{i j}$.

The Newman-Penrose scalar $\Psi_{4}$ is defined by

$$
\Psi_{4}=-C_{\alpha \beta \gamma \delta} n^{\alpha} \bar{m}^{\beta} n^{\gamma} \bar{m}^{\delta},
$$

where $C_{\alpha \beta \gamma \delta}$ represents the four-dimensional Weyl tensor (with the sign convention of [45]). For our simulations of vacuum spacetimes we assume that the Einstein equations are satisfied and calculate $\Psi_{4}$ from the Riemann tensor using $C_{\alpha \beta \gamma \delta}=R_{\alpha \beta \gamma \delta}$. The vectors $n$ and $\bar{m}$ form part of a null tetrad $l, n, m, \bar{m}$. Specifically, $l$ and $n$ denote ingoing and outgoing null vectors whereas the complex-valued $m$ is constructed out of two spatial vectors orthogonal to $l$ and $n$, such that

$$
-l \cdot n=1=m \cdot \bar{m},
$$

and all other inner products between the four-vectors vanish. $\Psi_{4}$ transforms as a spin-weight -2 field (that is, under tetrad rotations which leave $l$ and $n$ unchanged and rotate $m$ and $\bar{m}$ by an angle $\theta$, we have $\Psi_{4} \rightarrow e^{-2 i \theta} \Psi_{4}$ ). Such objects represent symmetric trace-free tensor fields on a sphere (in our case $R_{\alpha \beta \gamma \delta} n^{\alpha} n^{\gamma}$ ) in terms of a complex scalar field. For a quick introduction to spin-weighted fields see, e.g., [46].

In order to ensure that $\Psi_{4}$ represents a measure of the outgoing gravitational radiation, the tetrad vectors need to be chosen with care. Assuming that the spacetime metric is close to a Kerr metric, as is the case at sufficient distances from the black holes, the correct choice is the Kinnersley tetrad [47]. In numerical simulations, however, the exact Kinnersley tetrad is not available so that one needs to resort to approximations. Here we follow the traditional approach in numerical relativity and form the tetrad from the timelike unit normal field as well as applying the GramSchmidt orthonormalization procedure to the threedimensional vectors

$$
\begin{gathered}
u^{i}=[-y, x, 0], \quad v^{i}=[x, y, z], \\
w^{i}=g^{i a} \epsilon_{a b c} u^{a} v^{b} .
\end{gathered}
$$

This procedure, possibly with different choices in the order of the triad vectors in the orthonormalization, is a standard method in numerical relativity (see, e.g., [48]) and also forms the basis for the Lazarus method [49]. The tetrad vectors are then given by

$$
n^{0}=\frac{1}{\sqrt{2} \alpha} \quad n^{i}=\frac{1}{\sqrt{2}}\left(\frac{-\beta^{i}}{\alpha}-v^{i}\right)
$$

$$
\begin{array}{cc}
l^{0}=\frac{1}{\sqrt{2} \alpha} & l^{i}=\frac{1}{\sqrt{2}}\left(\frac{-\beta^{i}}{\alpha}+v^{i}\right), \\
m^{0}=0 & m^{i}=\frac{1}{\sqrt{2}}\left(w^{i}+i u^{i}\right) .
\end{array}
$$

Next, we need to express Eq. (29) in terms of the threedimensional quantities available on each time slice. This is achieved by virtue of the Gauss-Codazzi and the Mainardi equations which relate the spacetime projections of the four-dimensional Riemann tensor to its three-dimensional counterpart and the ADM variables according to

$$
\begin{gathered}
\perp R_{\alpha \beta \gamma \delta}=\mathcal{R}_{\alpha \beta \gamma \delta}+K_{\alpha \gamma} K_{\beta \delta}-K_{\alpha \delta} K_{\beta \gamma}, \\
\perp R_{\mu \beta \gamma \delta} \hat{n}^{\mu}=D_{\gamma} K_{\beta \delta}-D_{\delta} K_{\beta \gamma}, \\
\perp R_{\mu \beta \nu \delta} \hat{n}^{\mu} \hat{n}^{\nu}=\mathcal{R}_{\beta \delta}-K_{\beta \mu} K_{\delta}^{\mu}+K K_{\beta \delta},
\end{gathered}
$$

where $\mathcal{R}_{\alpha \beta \gamma \delta}$ is the three-dimensional Riemann tensor, $\hat{n}^{\alpha}$ the timelike unit normal vector associated with the foliation, and we follow York's [20] notation for the projection operator $\perp$, which is, for example, for an arbitrary tensor $T_{\alpha \beta}$,

$$
\perp T_{\alpha \beta}:=\left(\delta_{\alpha}^{\mu}+\hat{n}^{\mu} \hat{n}_{\alpha}\right)\left(\delta_{\beta}^{\nu}+\hat{n}^{\nu} \hat{n}_{\beta}\right) T_{\mu \nu} .
$$

In our coordinate basis adapted to the " $3+1$ " decomposition, we are thus able to express $\Psi_{4}$ exclusively in terms of the ADM variables as well as the triad vectors constructed from (31) according to

$$
\begin{aligned}
\Psi_{4}= & -\frac{1}{4}\left(R_{a b c d} v^{a} v^{c}-2 \perp R_{\alpha b c d} \hat{n}^{\alpha} v^{c}+\perp R_{\alpha b \gamma d} \hat{n}^{\alpha} \hat{n}^{\gamma}\right) \\
& \times\left(u^{b}-i w^{b}\right)\left(u^{d}-i w^{d}\right) .
\end{aligned}
$$

The contributions of the individual modes $\ell, m$ are obtained from projecting $\Psi_{4}$ onto the spherical harmonics $Y_{\ell m}^{-2}$ of spin weight -2 . These projections are defined in terms of the scalar product

$$
A_{\ell m}=\left\langle Y_{\ell m}^{-2}, \Psi_{4}\right\rangle=\int_{0}^{2 \pi} \int_{0}^{\pi} \Psi_{4} \overline{Y_{\ell m}^{-2}} \sin \theta d \theta d \phi
$$

which, in practice, is evaluated at a finite extraction radius $r_{\text {ext }}$.

The spin-weighted spherical harmonics $Y_{\ell m}^{s}$ can be defined in terms of the Wigner $d$ functions (e.g., [50]) as

$$
Y_{\ell m}^{s}(\theta, \varphi)=(-1)^{s} \sqrt{\frac{2 \ell+1}{4 \pi}} d_{m(-s)}^{\ell}(\theta) e^{i m \varphi},
$$

where

$$
\begin{aligned}
d_{m s}^{\ell}(\theta)= & \sum_{t=C_{1}}^{C_{2}} \frac{(-1)^{t}[(\ell+m) !(\ell-m) !(\ell+s) !(\ell-s) !]^{1 / 2}}{(\ell+m-t) !(\ell-s-t) ! t !(t+s-m) !} \\
& \times(\cos \theta / 2)^{2 \ell+m-s-2 t}(\sin \theta / 2)^{2 t+s-m},
\end{aligned}
$$

with $C_{1}=\max (0, m-s)$ and $C_{2}=\min (\ell+m, \ell-s)$. 
For $\ell=2$ and spin weight $s=-2$, we have

$$
\begin{aligned}
& Y_{2-2}^{-2}=\sqrt{\frac{5}{64 \pi}}(1-\cos \theta)^{2} e^{-2 i \phi}, \\
& Y_{2-1}^{-2}=\sqrt{\frac{5}{16 \pi}} \sin \theta(1-\cos \theta) e^{-i \phi}, \\
& Y_{20}^{-2}=\sqrt{\frac{15}{32 \pi}} \sin ^{2} \theta, \\
& Y_{21}^{-2}=\sqrt{\frac{5}{16 \pi}} \sin \theta(1+\cos \theta) e^{i \phi}, \\
& Y_{22}^{-2}=\sqrt{\frac{5}{64 \pi}}(1+\cos \theta)^{2} e^{2 i \phi} .
\end{aligned}
$$

In practice, the integrand on the right-hand side of Eq. (40) is evaluated on the Cartesian grid and interpolated onto a sphere of extraction radius $r_{\text {ext }}$ using fifth-order polynomials. The integration over the sphere is performed using the fourth-order Simpson method.

While this procedure is straightforward from a numerical point of view, we emphasize one delicate point. In order to reduce the computational costs, numerical simulations are often performed with explicit use of symmetry properties of the spacetime under consideration. For this purpose it is important to take into account the transformation of the variables under inversion of the $x, y$, or $z$ coordinate. In our case the nontrivial operation is the symmetry across the $x y$ plane. This problem manifests itself in the calculation of the modes according to (40) where the integrand is directly available only in the range $0 \leq \theta \leq \pi / 2$. Using the parity properties of the functions involved, however, we are able to transform the right-hand side of Eq. (40) into an integral restricted to the northern hemisphere. In particular, in the case of reflection in the $z$ direction $[(x, y, z) \rightarrow(x, y,-z)]$, which is relevant here, the real part of $\Psi_{4}$ behaves like an even function, whereas the imaginary part of $\Psi_{4}$ behaves as an odd function.

Similarly, the harmonics $Y_{22}^{-2}, Y_{2-2}^{-2}$ transform into the complex conjugates of each other. In summary,

$$
\begin{gathered}
\Psi_{4}(\pi-\theta, \phi)=\overline{\Psi_{4}(\theta, \phi),} \\
Y_{22}^{-2}(\pi-\theta, \phi)=\overline{Y_{2-2}^{-2}(\theta, \phi)}, \\
Y_{2-2}^{-2}(\pi-\theta, \phi)=\overline{Y_{22}^{-2}(\theta, \phi)} .
\end{gathered}
$$

We use the following relation valid for arbitrary functions of $\theta$

$$
\int_{0}^{\pi} f(\theta) d \theta=\int_{0}^{\pi / 2}[f(\theta)+f(\pi-\theta)] d \theta,
$$

and are thus able to calculate

$$
\begin{aligned}
A_{22} & =\left\langle Y_{22}^{-2}, \Psi_{4}\right\rangle=\int_{0}^{2 \pi} \int_{0}^{\pi} \Psi_{4} \overline{Y_{22}^{-2}} \sin \theta d \theta d \phi \\
& =\int_{0}^{2 \pi} \int_{0}^{\pi / 2}\left(\Psi_{4} \overline{Y_{22}^{-2}}+\overline{\Psi_{4}} Y_{2-2}^{-2}\right) \sin \theta d \theta d \phi .
\end{aligned}
$$

An equivalent change of basis to represent functions on the sphere has been discussed by Zlochower et al. in [51].

In the study of numerical simulations of black-hole binary systems, one is often interested in the amount of energy and momenta radiated away from the system in the form of gravitational radiation. In terms of the NewmanPenrose scalar $\Psi_{4}$, these are given by the expressions

$$
\begin{gathered}
\frac{d E}{d t}=\lim _{r \rightarrow \infty}\left[\frac{r^{2}}{16 \pi} \int_{\Omega}\left|\int_{-\infty}^{t} \Psi_{4} d \tilde{t}\right|^{2} d \Omega\right], \\
\frac{d P_{i}}{d t}=-\lim _{r \rightarrow \infty}\left[\frac{r^{2}}{16 \pi} \int_{\Omega} \ell_{i}\left|\int_{-\infty}^{t} \Psi_{4} d \tilde{t}\right|^{2} d \Omega\right], \\
\frac{d J_{z}}{d t}=-\lim _{r \rightarrow \infty}\left\{\frac { r ^ { 2 } } { 1 6 \pi } \operatorname { R e } \left[\int_{\Omega}\left(\partial_{\phi} \int_{-\infty}^{t} \Psi_{4} d \tilde{t}\right)\right.\right. \\
\left.\left.\times\left(\int_{-\infty}^{t} \int_{-\infty}^{\hat{t}} \bar{\Psi}_{4} d \tilde{t} d \hat{t}\right) d \Omega\right]\right\}
\end{gathered}
$$

(see, e.g., Ref. [52]), where

$$
\ell_{i}=(-\sin \theta \cos \phi,-\sin \theta \sin \phi,-\cos \theta) .
$$

We have listed these relations explicitly, because of different conventions in use in the literature. In particular we emphasize the difference by a factor of $1 / 4$ with Eqs. (22)(24) of [52] which arises out of differences in the scaling of the tetrad vectors [cf. our Eqs. (32) and (33) with their Eq. (30)].

The expression for the energy can be simplified by using the expansion of $\Psi_{4}$ in modes $\ell, m$. Taking into account the orthonormality of the spin-weighted harmonics we obtain

$$
\frac{d E}{d t}=\lim _{r \rightarrow \infty}\left[\frac{r^{2}}{16 \pi} \sum_{l, m}\left|\int_{-\infty}^{t} A_{\ell m} d \tilde{t}\right|^{2}\right] .
$$

In particular, this relation enables us to calculate the energy radiated in an individual mode. For the equal-mass systems considered in this work, we find $>98 \%$ of the energy to be radiated in the form of the dominant $\ell=2, m= \pm 2$ modes.

Finally, let us note that in analyzing waveform modes as functions of time, it is useful to split the complex function representing $r \Psi_{4}$ (or, say, the strain $h$ ) as

$$
r \Psi_{4}(t)=A(t) e^{i \varphi(t)}, \quad \omega(t)=\frac{\partial \varphi(t)}{\partial t},
$$

as suggested in [11]. In this paper this representation proves particularly useful to compare different initial data sets as in Sec. VII. 


\section{B. Total energy, linear and angular momentum}

In general relativity unambiguous notions of the energymomentum four-vector and angular momentum can only be assigned to a spacetime as global quantities, determined from the asymptotic structure of the spacetime. In this sense two types of quantity can be defined: those that are conserved by time evolution and those that decrease with time, expressing the radiation of energy momentum and angular momentum to infinity.

The expression for the energy momentum at spatial infinity, which is time independent and which corresponds to a four-vector under Lorentz transformations, was given first by Arnowitt, Deser and Misner in 1962 [53] in the context of the Hamiltonian formalism. This quantity is usually called the ADM energy momentum, the time component being called the ADM energy or, somewhat inconsistently, the ADM mass, different from the rest mass to be defined below. The expressions can be given as limits of surface integrals defined at finite radius, and are evaluated in asymptotically Cartesian (regular) coordinates $\left\{x^{i}\right\}$ where the components of the spatial metric tend to $\operatorname{diag}(1,1,1)$ for large radii. The surfaces are then taken as spheres $S_{r}$ of radius $r$.

We define the surface integrals (which we will also refer to as ADM integrals)

$$
\begin{gathered}
E(r)=\frac{1}{16 \pi} \int_{S_{r}} \sqrt{g} g^{i j} g^{k l}\left(g_{i k, j}-g_{i j, k}\right) d S_{l}, \\
P_{j}(r)=\frac{1}{8 \pi} \int_{S_{r}} \sqrt{g}\left(K_{j}^{i}-\delta_{j}^{i} K\right) d S_{i}, \\
J_{j}(r)=\frac{1}{8 \pi} \epsilon_{j l}^{m} \int_{S_{r}} \sqrt{g} x^{l}\left(K_{m}^{i}-K \delta_{m}^{i}\right) d S_{i},
\end{gathered}
$$

which have to be evaluated in an asymptotically Cartesian coordinate system.

The ADM energy $M_{\mathrm{ADM}}$ and linear and angular momentum $P_{j}$ and $J_{j}$ are then given by $[20,54,55]$

$$
\begin{gathered}
M_{\mathrm{ADM}}=\lim _{r \rightarrow \infty} E(r), \\
P_{j}=\lim _{r \rightarrow \infty} P_{j}(r), \\
J_{j}=\lim _{r \rightarrow \infty} J_{j}(r),
\end{gathered}
$$

and the rest mass $M_{R}$ can be defined as $M_{R}^{2}=M_{\mathrm{ADM}}^{2}-$ $\sum_{j=1,3} P_{j} P_{j}$.

For radiation processes we also require definitions of total energy, linear and angular momentum that decrease as energy and linear as well as angular momentum are radiated to infinity. The appropriate quantities are the Bondi quantities [56], which can be defined as taking the limit of the ADM integrals not toward spatial, but rather null, infinity [57-59], i.e., the limit to infinite distance is taken for constant retarded time instead of on a fixed Cauchy slice. In the context of our numerical treatment, the ADM and Bondi quantities can be computed rather accurately by computing values at several radii, and then performing a Richardson extrapolation (in extraction radius, not, as is more usual, in grid spacing) to infinity. Here the Bondi quantities can be computed at any time for a fixed extraction radius, and have to be compared between different radii by taking into account the light travel time between the timelike cylinders of different radii. This time delay can be estimated from a corresponding Schwarzschild solution as is done in [48] by the difference in the values of the radial "tortoise coordinate" values as

$$
\Delta T\left(R_{1}, R_{2}\right)=[R+2 M \ln (R / 2 M-1)]_{R=R_{1}}^{R=R_{2}},
$$

where the radii $R_{i}$ are understood as Schwarzschild radius (i.e., luminosity distance), and the Schwarzschild radius can be estimated from the simulation's radial coordinate $r$ by assuming it corresponds to the isotropic radial coordinate in Schwarzschild spacetime, which yields $R=r(1+$ $M / 2 r)^{2}$. We have however not applied this technique here, since a systematic study of wave-extraction techniques goes beyond the scope of this paper.

\section{NUMERICAL METHOD}

The numerical method of our black-hole simulations is based on a method of lines approach using finite differencing in space and explicit Runge-Kutta (RK) time stepping. For efficiency, Berger-Oliger-type adaptive mesh refinement (AMR) is used [60]. The new numerical results discussed in this paper were obtained with the BAM code $[6,15,16]$, which implements a particular AMR strategy that we describe below (we also compare with published results obtained with Sperhake's LEAN code [13]). Although BAM also includes an experimental oct-tree cell based algorithm that allows arbitrarily shaped refinement levels, this has not been used since a simpler box based algorithm is sufficient for black-holes binaries.

The numerical domain is represented by a hierarchy of nested Cartesian grids. The hierarchy consists of $L$ levels of refinement indexed by $l=0, \ldots, L-1$. A refinement level consists of one or more Cartesian grids with constant grid spacing $h_{l}$ on level $l$. A refinement factor of 2 is used such that $h_{l}=h_{0} / 2^{l}$. The grids are properly nested in that the coordinate extent of any grid at level $l, l>0$, is completely covered by the grids at level $l-1$. Of special interest are the resolutions $h_{\max }=h_{0}$ of the coarsest, outermost level, and $h_{\min }=h_{L-1}$ of the finest level.

Since we focus on the case of one or two black holes, a particularly simple grid structure is possible where each refinement level consists of exactly one or two nonoverlapping grids. While the size of these grids could be determined by truncation error estimates or by some field variable that indicates the need for refinement, for the purpose of convergence studies we have found it conve- 
nient to specify the size of the grids in advance. This allows, for example, the doubling of resolution within a predetermined coordinate range. Concretely, let $N_{l}$ be the number of points in any one direction for a cubical box with $N_{l}^{3}$ points on level $l$. On level $l$, center such a box on each of the black-hole punctures. If there are two punctures and the two boxes do not overlap, this is the layout that is used. If two boxes overlap, replace them by their bounding box, which is the smallest rectangular (in general noncubical) box that contains the two original boxes.

Assuming $N_{l}=N$ (a constant independent of $l$ ), a typical configuration around two punctures consists of two separate cubical boxes at $l=L-1$, but for decreasing $l$ and increasing $h_{l}$ the size of the boxes increases until starting at some intermediate level the boxes overlap and a single rectangular box is formed, which towards $l=0$ becomes more and more cubical.

The hierarchy of boxes evolves as the punctures move. We use the shift to track the position $x_{\text {punc }}^{i}$ of a puncture by integrating

$$
\partial_{t} x_{\text {punc }}^{i}=-\beta^{i}\left(x_{\text {punc }}^{j}\right),
$$

cf. [4], using the iterative Crank-Nicholson (ICN) method. The outermost box on level 0 and also several of the next finer levels are chosen to be single cubes of fixed size centered on the origin to avoid unnecessary grid motion.

Note that as long as one neglects the propagation of gravitational waves, the nesting described above represents in a natural manner the $1 / r$ falloff of the metric for a single puncture. For a single puncture and fixed $N$, doubling the grid spacing going from level $l$ to $l-1$, i.e., $h_{l-1}=2 h_{l}$, puts the boundary of a centered cube twice as far away. If a resolution of $h_{l}$ is sufficient to resolve the metric at $1 / r$, then $2 h_{l}$ should be sufficient to resolve the metric at $1 /(2 r)$ since this is the slowest falloff of any metric variable. This was the rationale for the nested box fixed mesh refinement (FMR) introduced in [15], which was found to work well in practice for the first 3D mesh-refinement evolutions of black holes [16]. This FMR nesting strategy generalizes straightforwardly to the case of two moving punctures as outlined above.

In the presence of gravitational waves further demands for spatial resolution arise: the wave amplitude falls off with $1 / r$, corresponding to the roughly constant amplitude of the "predicted" signal $r \psi_{4}$, while the wavelength is approximately constant. The spatial profile of the signal thus requires constant radial resolution with increasing distance, while the amplitude falloff leads to increasing accuracy requirements as distance increases, in order to separate the waves from the background. Correspondingly, the grids need to be adapted when waves need to be traced accurately to typical wave-extraction distances, which in a setup as presented here are still rather limited by computational cost. In actual runs it is thus convenient to use at least two different values for the $N_{l}$, one for the cubes that resolve the neighborhood of the punctures, another one for the levels where the wave extraction is performed. For Berger-Oliger time stepping most of the computational work is performed on the finest levels, so one chooses $N_{L-1}$ as small as possible (while still covering the entire black hole with sufficiently fine resolution), and we can gain some extra resolution for wave extraction at small extra cost by using a larger box for the levels on which waves are extracted.

The grids are cell centered. For example, in one dimension for the cell given by the interval $\left[0, h_{0}\right]$, the data on level 0 is located at the point $h_{0} / 2$, on level 1 at $h_{0} / 4$ and $3 h_{0} / 4$, on level 2 at $h_{0} / 8,3 h_{0} / 8,5 h_{0} / 8$, and $7 h_{0} / 8$, and so forth. Data are transferred between levels by polynomial interpolation using fifth-order polynomials for $O\left(h^{6}\right)$ errors, where the three-dimensional interpolant is obtained by successive one-dimensional interpolations.

On any given box with resolution $h_{l}$, we implement fourth-order finite differencing for the spatial derivatives of the Einstein equations. Standard centered stencils are used for all first- and second-order derivatives except for advection derivatives, $\beta^{i} \partial_{i}$. For second-order finite differencing, the advection terms required one-sided differencing for stability. For fourth-order finite differencing, we found that both centered and one-sided differencing can lead to severe instabilities with ICN time stepping, while "lopsided" stencils lead to stable evolutions (as suggested in [61]). Our runs are performed using such lopsided advection derivatives with fourth-order Runge-Kutta (RK4).

The code allows us to add artificial dissipation terms to the right-hand sides of the time evolution equations, schematically written as

$$
\partial_{t} \mathbf{u} \rightarrow \partial_{t} \mathbf{u}+Q \mathbf{u},
$$

in particular we use the standard Kreiss-Oliger dissipation $[62,63]$ operator $(Q)$ of order $2 r$

$$
Q=\sigma(-h)^{2 r-1}\left(D_{+}\right)^{r} \rho\left(D_{-}\right)^{r} / 2^{2 r},
$$

for a $(2 r-2)$-accurate scheme, with $\sigma$ a parameter regulating the strength of the dissipation, and $\rho$ a weight function that we currently set to unity. Adding artificial dissipation is apparently not required for stability in our runs, but we have used dissipation for RK4 evolutions to avoid high-frequency noise from mesh-refinement boundaries. We find that the inherently stronger dissipation of the ICN algorithm also rather efficiently suppresses noise from refinement boundaries, and our ICN test runs suggest that in this case the addition of Kreiss-Oliger dissipation is superfluous.

All AMR results for two punctures reported so far are based on codes that involve at least some second-order component, while BAM in principle allows fully fourthorder AMR. In particular, we apply sixth-order polynomial interpolation in space between different refinement levels 
so that all spatial operations of the AMR method are at least fourth-order. However, there are three sources of second-order errors. One is the initial-data solver, although this initial error appears to be negligible in the cases we consider here. Another source of second-order error is the implementation of the radiative boundary condition. However, the nested boxes position the outer boundary at sufficiently large distances such that these errors do not contribute significantly (ideally because they are causally disconnected from the wave-extraction zone). The final source of second-order error in our current runs is due to interpolation in time within the Berger-Oliger time stepping scheme, which is worth discussing in some more detail.

Berger-Oliger time stepping can be stated as recursive pseudocode for example as

evolve hierarchy $(l, \Delta t)$

evolve $(l, \Delta t)$

if $(l+1<L)$

evolve hierarchy $(l+1, \Delta t / 2)$

evolve hierarchy $(l+1, \Delta t / 2)$

if $(l>0)$

restrict_prolong $(l-1, l)$

$\operatorname{regrid}(l)$

The recursion is started by calling the function "evolve hierarchy" for $l=0$, i.e., beginning with the coarsest level. The function evolve_hierarchy evolves all levels from $l$ to $L-1$, the finest level, by a time step of $\Delta t$ forward in time. First, level $l$ is evolved by $\Delta t$ by the function "evolve." Then the function evolve_hierarchy calls itself recursively to advance level $l+1$ and all its sublevels twice by $\Delta t / 2$. The recursion ends if level $l+1$ does not exist, i.e., if $l+1$ is not less than $L$, then evolve_hierarchy does not call itself again. Once all levels $l$ through $L-1$ have reached the next time level, information is exchanged between levels $l-1$ and $l$, denoted by a call to "restrict_prolong," if $l>0$. In particular, the refinement boundary of $l$ is populated using information from $l-1$. The result is the new level $l$. Finally, the refinement hierarchy is updated by the function "regrid."

Although the time stepping used for evolution is fourthorder Runge-Kutta, there arises the additional issue of how to provide boundary values for the intermediate time levels of the Berger-Oliger algorithm that are not aligned in time with a coarser level (otherwise spatial interpolation can be used). There are several options for fourth-order boundaries.

The original suggestion by Berger and Oliger is to interpolate in time (over several coarse levels at different instances of time) in order to obtain boundary values for a fine level. One can use three time levels of the coarser level to perform quadratic interpolation (third-order in the time step) resulting in overall second-order convergence when using a leapfrog scheme, e.g., as done in [15]. However, the convergence order and the stability of the algorithm de- pend on the form chosen for the Einstein equations and on the time-stepping algorithm used. For example, quadratic interpolation for ICN and a first-order in time, secondorder in space formulation can lead to a drop of convergence order and instabilities; see Schnetter et al. [64]. Other authors report success with different variants of time interpolation, e.g., [65,66].

An alternative approach is to replace the single point refinement boundary by a buffer zone consisting of several points, e.g., $[64,67,68]$. The buffer zone approach can be expected to perform well for the transmission of waves through refinement boundaries, see, e.g., [67] (note that special methods like [66] seem to achieve similar performance). The optimal number of buffer points is method dependent. For example, RK4 requires 4 source evaluations, and if the lopsided stencil with 3 points in one direction is used, then the numerical domain of dependence for a given point has a radius of 12 points. Therefore, it is possible to provide 12 buffer points at the refinement boundary and to perform one RK4 time step with size 3 stencils that does not require any boundary updates. Only after the time step is completed, the buffer zones have to be repopulated. In the context of Berger-Oliger AMR, the buffer update is based on interpolation from the coarser levels. Since every second time step at level $l$ coincides in time with level $l-1$, one can provide 24 buffer points, perform two time steps, and then update the buffer by interpolation in space. With 12 buffer points, one can interpolate in time to obtain data for the buffer points at intermediate time levels.

For the simulations reported here, our standard setup is to use RK4 with dissipation and lopsided advection stencils, 6 buffer points, quadratic interpolation in time, and Berger-Oliger time stepping on all but the outermost grids. Let us comment on these choices.

For some grid configurations we have encountered instabilities for very large, coarse grids, that experimentally are connected to the large time steps on the coarse grid. We were able to cure these instabilities by turning-off BergerOliger time stepping for the outermost grids (cf. [6] where this idea was introduced in a different context).

To use fewer than 12 buffer points, we can interpolate into all buffer points before starting a RK4 update as described, and then evolve all points except the outermost points located exactly on the boundary, which are kept fixed at their initial interpolated value. The inner points next to the boundary are updated using second-order finite differencing for the centered derivatives. The advection derivatives near the boundary are computed by shifted fourth-order stencils, where the shift is determined such that the stencil fits onto the grid while maintaining the desired one-sidedness as far as possible. Experimentally, using just 6 buffer points leads to very small differences compared to 12 buffer points, however smaller buffer zones lead to noticeable differences. Even though for large 
grids the number of buffer zones becomes negligible, for the grid sizes that we have to use, the buffer points impact the volume of the grids significantly. For example, for a box of size 64 in one direction, adding 6 points on both sides instead of 12 points corresponds to a savings of $35 \%$ in the total number of points. For clarity, we always quote grid sizes without buffer points, because this is the number of points owned by a particular grid.

Using quadratic interpolation in time is, apart from the outer boundary treatment, the only source of second-order errors in the evolution scheme. We checked for a few cases that running without Berger-Oliger time stepping entirely led to only small differences compared to other error sources. However, for sufficiently high resolutions, quadratic interpolation in time should become the dominant error. In principle, we can resolve this issue by either not using Berger-Oliger time stepping or by using larger buffer zones, which at the moment is prohibitively expensive in resources.

We have also experimented with higher order in time interpolation, although a systematic analytical and numerical analysis beyond these first experiments is needed. Simply using additional coarse time levels was not successful. In general, if at time $t$ a fine level $l$ is not aligned in time with the coarser level $l-1$, we use the grid functions on level $l-1$ at different times to interpolate to time $t$. For quadratic interpolation these different times are $t+\Delta t, t-$ $\Delta t$, and $t-3 \Delta t$, where $2 \Delta t$ is the time step on level $l-1$. As mentioned before this kind of interpolation leads to overall second-order accuracy in time at the interpolated points. We routinely use this approach and it leads to stable evolutions. In order to obtain a fully fourth-order scheme we have included additional coarse levels at times $t-5 \Delta t$ and $t-7 \Delta t$. However, this extended interpolation scheme over five different times leads to oscillations at the refinement boundaries, which are the points where we use interpolation in time. These oscillations increase with resolution and are thus likely instabilities which would cause the code to fail at sufficiently high resolution. At the resolution considered in this paper these instabilities do not cause the code to fail. However, they are a significant source of noise, which propagates out of the refinement boundaries into the rest of the grid. Since this noise is not convergent, it eventually spoils convergence in the entire grid. One reason for this problem may be the high degree of asymmetry in the interpolation stencil which uses four points before time $t$ and only one after $t$.

Finally, we note that BAM is message passing interface parallelized. The dynamic grid hierarchy with moving and varying boxes introduces an additional communication overhead compared to the FMR runs that BAM was used for previously [6]. For up to 128 processors scaling seems reasonable for a constant problem size per processor, but we do expect issues for larger processor numbers, which we have not been able to test yet.

\section{SINGLE PUNCTURE WITH DYNAMIC CONFORMAL FACTOR}

\section{A. Numerical experiments for a single stationary puncture}

In this section we apply the $\phi$ and $\chi$ moving-puncture methods to evolutions of a single Schwarzschild puncture. This provides an excellent test case, because we can compare with the analytic results in [34], and study the convergence properties of the code without the added complication of moving mesh-refinement boxes.

The initial data are as described in Sec. II A, where $u=$ 0 and $\tilde{A}_{i j}=0$ on the initial slice, and we choose $m_{1}=$ $M=1$. We use a "precollapsed" lapse of $\alpha=\psi_{0}^{-2}$ for these runs, but stress that similar convergence properties are found with an initial lapse of $\alpha=1$. The convergence series consists of evolutions with $N=64^{3}, 96^{3}, 128^{3}$ grid points in each box, and seven levels of refinement below the coarsest level (making a total of eight levels). The resolutions on the coarsest levels are $h_{\max }=6 M, 4 M$, $3 M$, and the resolutions on the finest levels and at the puncture are $h_{\min }=6 M / 128,4 M / 128,3 M / 128$. The gauge choice is $t t t$, and $\eta=2.0 / M$. For these runs (and only for these) a uniform time step was used on all levels (i.e., not Berger-Oliger) in order to fully test the fourthorder accuracy of the code.

As discussed in [34], a $1+\log$ evolution of Schwarzschild reaches a stationary slice, and at the puncture $\beta^{2}=\beta_{i} \beta^{i}=0.5239$ the Schwarzschild radial coordinate is $R=1.3124 M$. After $50 M$ of evolution, these values are reached to within $1.3 \%$ and $0.5 \%$ in the highest resolution runs using the $\phi$ method. With the $\chi$ method, the errors in $\beta^{2}$ and $R$ are $0.6 \%$ and $0.2 \%$. Figure 1 shows several of the BSSN variables after $50 M$ of evolution with the $\chi$ method.

The convergence of the $\phi$ method is demonstrated in Fig. 2, which shows convergence plots of $\tilde{g}_{x x}, \phi, \alpha, \beta^{y}$, and the Hamiltonian and momentum constraints. The data are taken along the $y$ axis at $t=50 M$ on the finest level of the mesh-refinement scheme. The errors in the Hamiltonian and momentum constraints cover a wide range, so the logarithm of the scaled errors is shown. The differences are scaled assuming fourth-order convergence, and the code demonstrates good fourth-order convergence everywhere except at the points closest to the puncture.

The $\phi$ variable shows extremely poor convergence at the puncture, but this is to be expected: $\phi$ diverges like $\ln (r)$ near the puncture. What is remarkable is that this nonconvergent behavior remains localized at the puncture, and does not affect the accuracy or stability of the evolution as a whole.

Figure 3 shows similar convergence plots for the $\chi$ method. In this case the $\chi$ variable, which should behave like $r^{2}$ near the puncture, is seen to converge everywhere. The constraints and $\beta^{y}$ also show better convergence prop- 

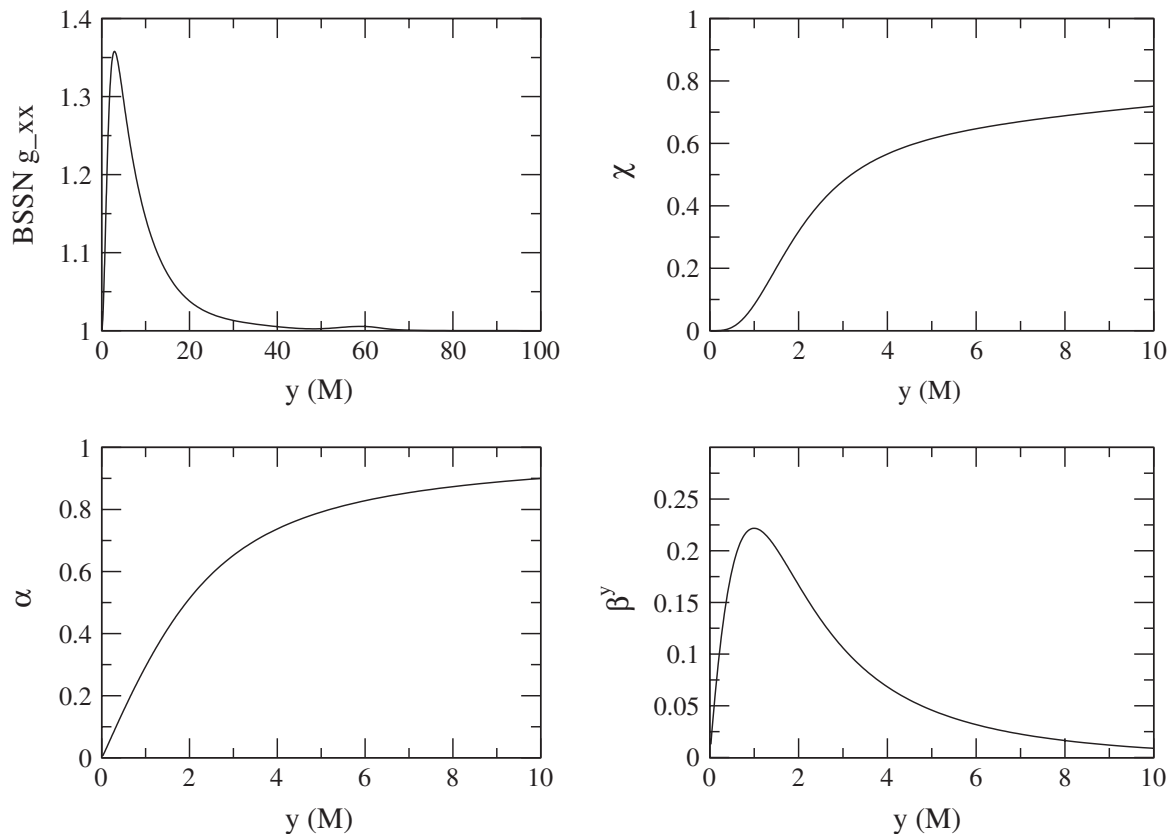

FIG. 1. The BSSN variables $\tilde{g}_{x x}, \chi, \alpha$, and $\beta^{y}$ after $50 M$ of evolution of a Schwarzschild puncture using the $\chi$ method. A small pulse due to the initial adjustment of the gauge can be seen at about $y=60 M$ in $\tilde{g}_{x x}$. The main features of the other variables are confined to $y<10 M$.
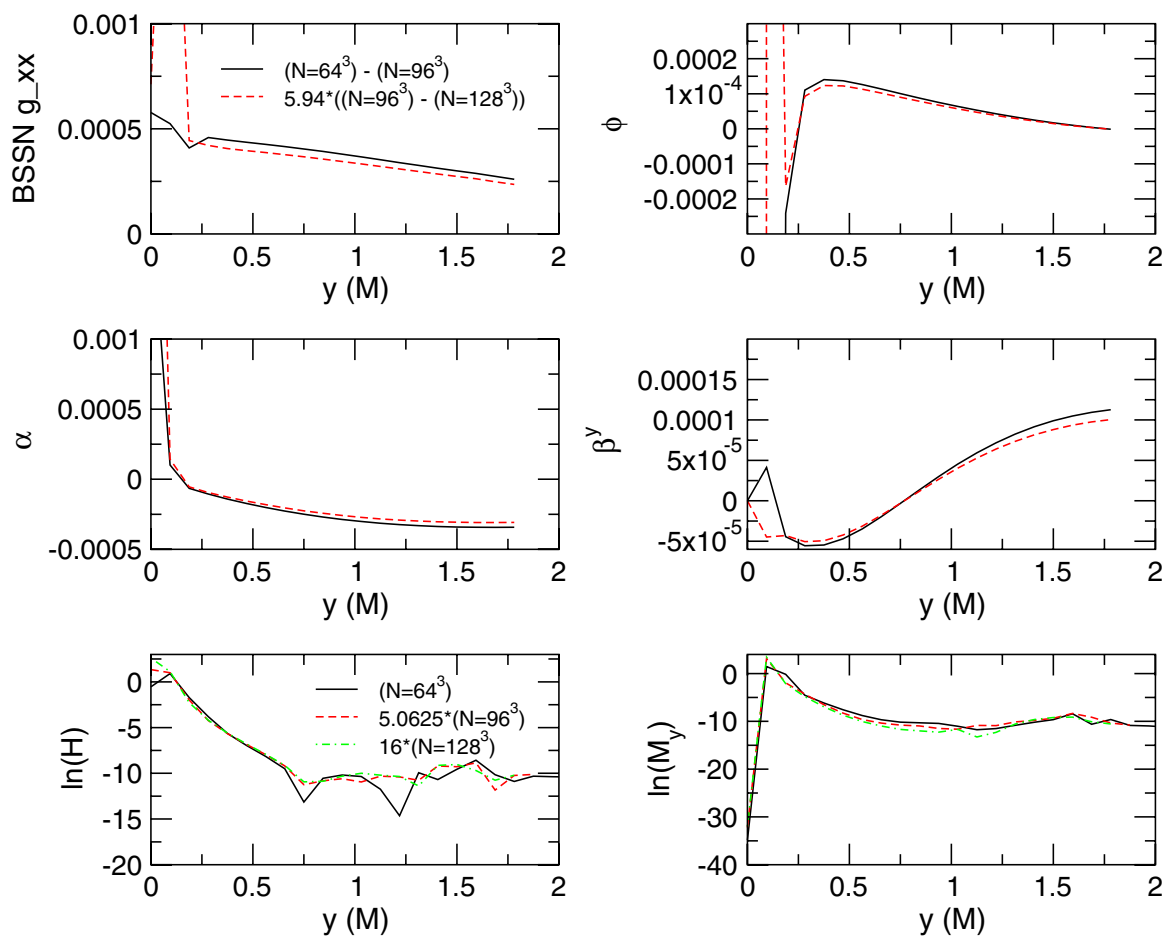

FIG. 2 (color online). Fourth-order convergence of a Schwarzschild puncture after $50 M$ of evolution, using the $\phi$ method. Results were taken from runs with $N=64^{3}, 96^{3}$ and $128^{3}$ points with octant symmetry. The plots show the differences between the three runs, scaled to be consistent with fourth-order accuracy. For the Hamiltonian constraint and $y$ component of the momentum constraint, which should converge to zero, we show the logarithm of the scaled values. 

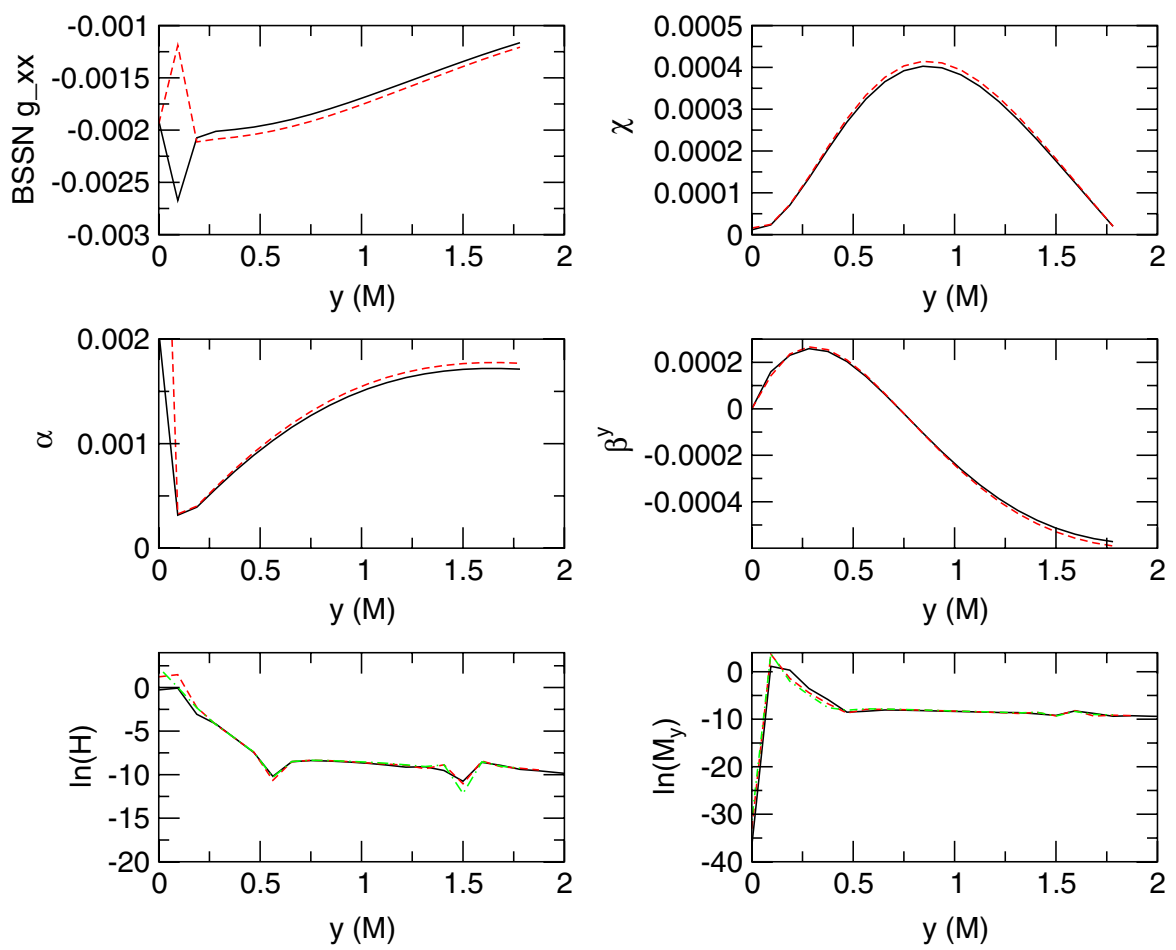

FIG. 3 (color online). Fourth-order convergence of a Schwarzschild puncture after $50 M$ of evolution, using the $\chi$ method. The parameters match those used for the runs discussed in Fig. 2.

erties near the puncture. This is consistent with the comparison with the stationary $1+\log$ solution, where we see that the $\chi$ method was more accurate at the puncture.

We draw three conclusions from these results. (1) Our code is fourth-order accurate for the resolutions used in this work, at least when the mesh-refinement boxes do not move and a uniform time step is used. (2) The movingpuncture method extremely accurately reaches the stationary $1+\log$ slicing, and, since the puncture no longer represents a second infinity, the solution is well resolved up to the puncture. (3) Both the $\phi$ and $\chi$ methods are stable and accurate, but the $\chi$ method shows (as expected) better convergence properties at the puncture. As a test of the stability of the method at extremely high resolutions, we have also evolved a Schwarzschild puncture with resolutions of up to $M / 512$ at the puncture for $100 M$ and saw no signs of instability.

In addition, we emphasize that the only variable that diverges at the puncture is $\phi$. When the $\chi$ method is used, all variables are finite at the puncture. Some variables are discontinuous at the puncture. This leads to incorrect evaluation of finite-difference derivatives at the grid points closest to the puncture (the number of points depends on the width of the finite-difference stencil used), but these errors do not seem to propagate away from the puncture, and spoil the convergence of the variables in question only near the puncture. These errors could presumably be reduced or removed by using appropriate one-sided derivatives next to the puncture, but we have obtained sufficiently accurate results without need of such a sophisticated treatment.

\section{Coordinate dependence on $\eta$}

The geometry of the stationary $1+\log$ slice is unique, but the coordinates of that final slice are not. One quantity that alters the final coordinates is the gamma-freezing damping parameter, $\eta$. The parameter $\eta$ was originally introduced in [30] for fixed-puncture evolutions to prevent oscillations in the shift vector as well as long-term drifts in the metric variables. The effect of $\eta$ in our new evolutions is demonstrated in Fig. 4, which shows the coordinate location of the Schwarzschild horizon $R=2 M$ after $50 M$ of evolution, as a function of $\eta$. We see that the coordinate size of the black hole differs by more than a factor of 2 between $\eta=0$ and $\eta=3 / M$; similar effects were alluded to in [12]. As a result, different choices of $\eta$ correspond to different effective numerical resolutions across the black hole. For example, with $\eta=0$ and a central resolution of $M / 16$, there are about 26 grid points across the interior of the black hole. With $\eta=3 / M$, there are about 59 grid points across the black hole - it is resolved twice as well. On the other hand, if the finest box in the mesh-refinement structure contains $32^{3}$ points, then this box contains the entire black hole when $\eta=0$, but does not when $\eta>$ $1.0 / M$.

In any black-hole simulation, one must decide which is more important, the effective finest resolution or the effective size of the finest box. Perhaps more importantly, the 

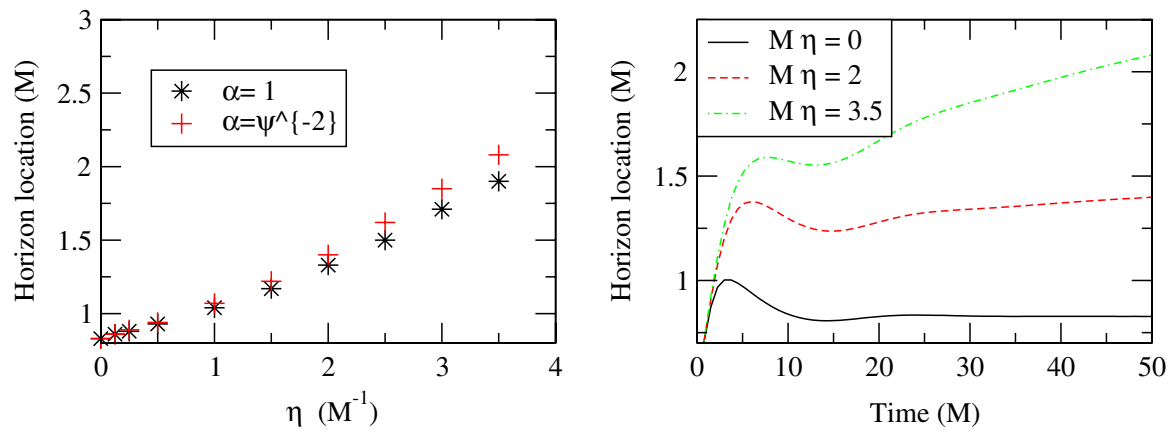

FIG. 4 (color online). Left: Coordinate location of $R=2 M$ after $50 M$ of evolution, as a function of the damping parameter, $\eta$, with initial lapse $\alpha=1$ and $\alpha=\psi^{-2}$. Right: Coordinate location $R=2 M$ as a function of time, for $M \eta=0,2.0,3.5$, using initial $\alpha=1$.

effect of $\eta$ on the coordinates shows that one must be careful when comparing runs that use different resolutions and/or box sizes, and different values of $\eta$.

Larger values of $\eta$ also cause a larger drift in the horizon location with time. Although the geometry becomes stationary after about $40 \mathrm{M}$ of evolution, the numerical coordinates may not. This is clear from the right-hand panel of Fig. 4. We see that, if we wish to minimize the drift in the numerical coordinates, lower values of $\eta$ are better. We will see similar results in Sec. VI in the case of black-hole binaries.

\section{B. Numerical experiments for a single spinning puncture}

We now look at results for evolutions of a single spinning puncture. These allow us to test the moving-puncture method for spinning black holes, and provide a nontrivial test of the wave-extraction algorithm for a black-hole spacetime. The initial data are now based on the BowenYork extrinsic curvature for a single black hole with nonzero spin, which can be considered as a Kerr black hole plus Brill-wave radiation [22,69-71]. In an evolution the

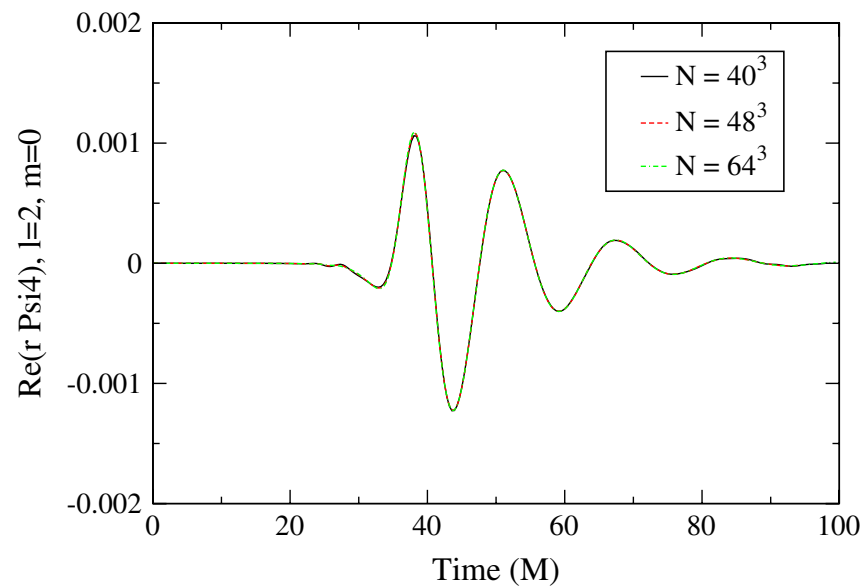

FIG. 5 (color online). Real part of the $l=2, m=0$ mode of $r \Psi_{4}$, extracted at $r=30 m_{1}$, for a simulation of a single spinning Bowen-York puncture with $S=0.2$. additional radiation will leave the system, and only the Kerr black hole will remain. The energy of the radiation has been estimated in the past by studying the initial data $[69,70]$, with a radiation content of up to $3 \%$ for a nearmaximally spinning Kerr black hole.

We considered a Bowen-York puncture with mass parameter $m_{1}=1$ and angular momentum parameter $S_{z}=$ $0.2 m_{1}^{2}$. As discussed in Sec. II A the mass of the black hole can be estimated using Eq. (6). For these data, the blackhole mass is $M=1.0155 m_{1}$. The Kerr parameter can then be estimated as $a=s / M^{2}=0.194$.

The spinning puncture was evolved for $100 m_{1}$ using the $\phi$ and $\chi$ methods. Convergence tests consisted of runs with seven levels of refinement, box sizes of $40^{3}, 48^{3}$, and $64^{3}$ points, and resolutions of the coarsest box of $h_{\max }=6 m_{1}$, $5 m_{1}, 3.75 m_{1}$. The $(l=2, m=0)$ mode of $r \Psi_{4}$ for these three simulations is shown in Fig. 5.

Figure 6 shows convergence plots for the $\phi$ and $\chi$ methods. We have plotted the differences in $\operatorname{Re}\left(r \Psi_{4}\right)_{20}$ between the three grid sizes, and scaled the medium-fine

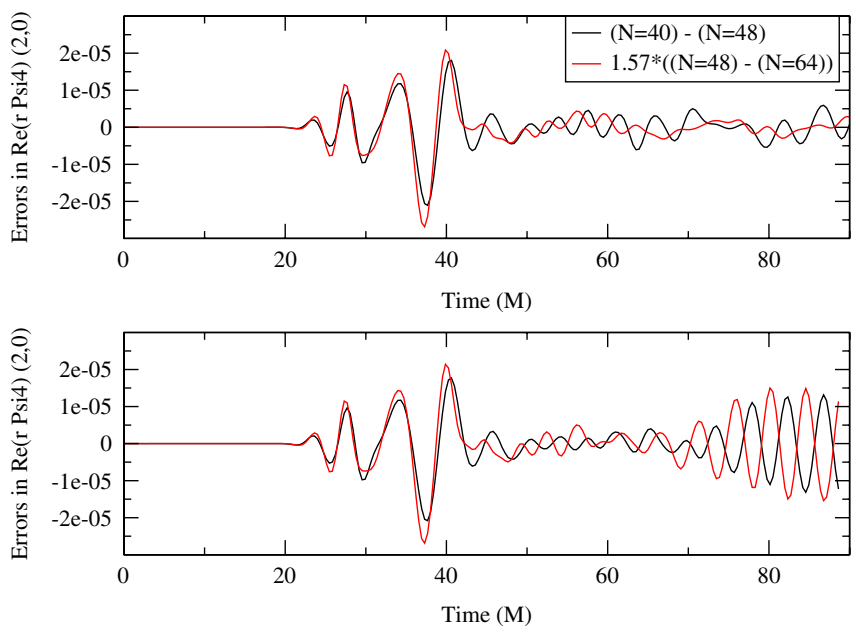

FIG. 6 (color online). Errors in the real part of the $l=2, m=$ 0 mode of $r \Psi_{4}$, extracted at $r=30 m_{1}$ for the same simulations as in Fig. 5. The upper plot shows results from runs with the $\phi$ method, while the lower plot shows results with the $\chi$ method. See text for the grid details and discussion. 
difference by a factor of 1.57 , consistent with fourth-order convergence. Both methods show reasonable fourth-order convergence for the first $\sim 40 \mathrm{M}$ of evolution, demonstrating that the wave-extraction algorithm is fourth-order convergent. Convergence in the waveform (and the evolution variables) is lost after that time. This may be due to reflections from mesh-refinement boundaries. However, for both the $\phi$ and $\chi$ runs the errors are extremely small, and of comparable magnitude.

\section{NUMERICAL EXPERIMENTS FOR TWO ORBITING PUNCTURES}

In this section we calibrate our code for binary evolutions. These will be the principal application of our code in future work, and we therefore perform a more detailed study than in the case of single black holes. We focus on runs that use the initial-data parameters of the run "R1" in [11], for which comparison simulations were also performed in [13]. We evolve these data with both the $\phi$ and $\chi$ variants of the moving-puncture method, and in each case compare runs with $\eta=1$ and $\eta=2$, to determine which aspects of the simulations are most strongly affected by different gauge choices.

The main goal of this paper is to demonstrate the accuracy and efficiency of our code, and of course to verify that it gives correct results. We begin by determining the grid setups necessary to achieve fourth-order-accurate results, and present our results with error bars calculated from the difference between the highest resolution runs and Richardson-extrapolated values. By "grid setup" we do not simply mean "resolution"; the sizes of the meshrefinement boxes are also important, both for the accuracy of the simulation, and the extracted physical quantities. Having done that, we compare our extracted waveforms with those produced by the independent LEAN code [13]. This is an extremely strong test: it validates both codes, in the sense that agreement suggests that any hidden coding errors have at most a negligible effect on the results, and also demonstrates the high accuracy of their results. Finally, we study in detail the accuracy of various extracted physical quantities (radiated energy, angular momentum, and angular frequency during inspiral), and their dependence on the radiation extraction radius and the gauge parameter $\eta$.

\section{A. Setup}

The initial-data parameters for the runs in this section are as follows. The punctures have mass parameters $m_{1}=$ $m_{2}=0.483$ and are placed on the $y$ axis at $y= \pm 3.257$ with momenta $p_{x}=\mp 0.133$. The individual black-hole masses, as determined by Eq. (6), are $M_{1}=M_{2}=0.505$, and the total $\mathrm{ADM}$ mass of the spacetime is $E_{\mathrm{ADM}}=$ 0.996. These parameters correspond to the run R1 in [11]. They result in $\sim 1.8$ orbits before merger at roughly 160M. (Recall that $M=\sum_{i} M_{i}$, as described in Sec. II A.)
We define three times indicating the merger time: $t_{A H}$, the time when an apparent horizon first forms, $t_{\alpha}$, the time at which the lapse at the center drops below the value 0.3 (following, e.g., $[11,72]$ ), and $t_{\max }$, the time at which $\left|\Psi_{4}\right|$ reaches a maximum (which depends on the extraction radius $r_{\text {ext }}$ ). While $t_{A H}$ is of immediate relevance regarding the simulation, it is also more costly to evaluate accurately, while accurate evaluation is trivial for $t_{\alpha}$ and $t_{\max }$. We therefore find it very useful to check convergence in phase by evaluating $t_{\alpha}$ and $t_{\max }$, and note that $t_{\alpha}$ and $\left(t_{\max }-r_{\text {ext }}\right)$ give an estimate for $t_{A H}$ which is accurate to a few $M$.

Note that in this section, all distances and times are either scaled with respect to the total black-hole mass, $M$ (consistent with our discussion of single black holes in Sec. V), in which case the appropriate unit is given [e.g., $r \Psi_{4}\left(M^{-1}\right)$ ], or, when no rescaling has been done, the numerical coordinates are used (e.g., "extraction at $r=30 ")$.

We label the grid setups for orbit runs with the notation $X\left[n_{1} \times N_{1}: n_{2} \times N_{2}:\right.$ buf $]\left[h_{\min }^{-1}: h_{\max }\right]$, where $X$ denotes the choice of conformal factor $\phi$ or $\chi$, and the grid is composed of $n_{1}$ levels of $N_{1}^{3}$ grid points and $n_{2}$ levels of $N_{2}^{3}$ grid points (reducing the number of grid points appropriately when discrete symmetries are applied), and buf mesh refinement buffer points are used. The quantities $h_{\min }$ and $h_{\max }$ denote the grid spacing on the finest and coarsest levels. The qualifier $X$ occasionally carries subscripts specifying further parameters. Examples would be $\phi[5 \times$ $32: 5 \times 64: 6][38.4: 8] \quad$ or $\quad \chi_{\eta=0.05}[5 \times 32: 5 \times 64: 6] \times$ [38.4:8]. The ratio of grid spacings between neighboring levels is always two.

We have performed a large number of runs, both complete convergence series and lower resolution "exploration runs" with different grid layouts, gauges, or numerical methods - for the presentation here we have to make a selection and present results from three series of runs, which we have found typical:

(1) $\operatorname{BAM} \phi 1: \phi_{\eta=1}[4 \times i: 6 \times 2 i: 6]$.

(2) $\operatorname{BAM} \chi 1: \chi_{\eta=1}[5 \times i: 4 \times 2 i: 6]$.

(3) $\operatorname{BAM} \chi 2: \chi_{\eta=2}[5 \times i: 4 \times 2 i: 6]$, i.e., as above, but with $\eta=2$.

The BAM $\phi 1$ series is representative of our early experiments. Apart from using the $\phi$ evolution variable, they also used the $t t t$ gauge advection choice. We later found the $\mathrm{BAM} \chi$ runs to be more accurate. In addition, the merger times converged from below, and convergence behavior was monotonic even at low resolutions. For the runs presented here, we see no strong difference between the $t t t$ and 000 gauge advection choices as described in Sec. II D. However, the manifestly strong hyperbolicity of the 000 gauge [37] makes that choice more attractive. For the $t t t$ choice, the slow-speed modes described in [36] can be clearly seen in animations of the grid variables. Both choices yield stable evolutions, but we regard the 000 choice as superior and have used it in the BAM $\chi$ runs presented here and in subsequent work. 
The runs presented here have 9 and 10 refinement levels (labeled from 0 for the coarsest to 8 or 9), and use twice the number of grid points on the outer levels than on the finest levels, as detailed in Table I. We find that this setup yields higher accuracy for wave extraction without too drastic an increase in computational cost. Typical performance numbers of our code are displayed in Table II. All runs are carried out with the symmetry $(x, y, z) \rightarrow(-x,-y, z)$ and $(x, y, z) \rightarrow(x, y,-z)$, reducing the computational cost by a factor of 4 compared with runs that do not exploit any discrete symmetries. The Courant factor $C=\Delta t / h_{l}$ is kept constant, and is set to $C=1 / 2$ for the inner grids, while for the outer grids at levels $0-2$ the time step is kept constant at the value of level 3. All runs presented here use the RK4 time integration scheme. Using the ICN scheme (without artificial dissipation) did not change results significantly. We find that for a constant Courant factor we occasionally encounter numerical instabilities in the outer regions of the simulation domain, but these were cured by freezing the size of the time step in the outermost 3 (BAM $\chi$ runs) or 4 (BAM $\phi 1$ run) levels.

All the BAM runs presented here use six AMR buffer points (see Sec. IV), which is less than required to isolate the fine level "half" time step from time interpolation errors at the mesh-refinement boundary, and, in particular, also less than required for the fully fourth-order Christmastree scheme suggested in [67]. We have experimented with using higher numbers of buffer points up to the number required for the Christmas-tree scheme, but have not found significant improvements in the results, which is consistent with the fact that we find fourth-order convergence and no significant improvement of the results when decreasing the time step. We conclude that at the resolutions presented here, six buffer points are enough to suppress errors from interpolation in time at mesh-refinement boundaries below the relevant threshold as far as the dynamics and low

TABLE I. Grid setups used for binary evolutions. See text for definition of the notation in the "Run" column. $h_{\min }$ and $h_{\max }$ (rounded to 3 digits) denote the finest and coarsest grid spacings, and $r_{\max }$ is the location of the outer boundary (rounded to 4 digits).

\begin{tabular}{lccc}
\hline \hline Run & $h_{\min }$ & $h_{\max }$ & $r_{\max }$ \\
\hline$\phi_{\eta=1}[4 \times 32: 6 \times 64: 6]$ & $1 / 25.6$ & 20 & 648.0 \\
$\phi_{\eta=1}[4 \times 40: 6 \times 80: 6]$ & $1 / 32.0$ & 16 & 672.0 \\
$\phi_{\eta=1}[4 \times 48: 6 \times 96: 6]$ & $1 / 38.4$ & $40 / 3$ & 666.7 \\
$\phi_{\eta=1}[4 \times 64: 6 \times 128: 6]$ & $1 / 51.2$ & 10 & 680.0 \\
$\phi_{\eta=1}[4 \times 72: 6 \times 144: 6]$ & $1 / 57.6$ & $80 / 9$ & 644.4 \\
$\chi_{\eta=1,2}[5 \times 32: 4 \times 64: 6]$ & $1 / 25.6$ & 10 & 325.0 \\
$\chi_{\eta=1,2}[5 \times 40: 4 \times 80: 6]$ & $1 / 32.0$ & 8 & 324.0 \\
$\chi_{\eta=1,2}[5 \times 48: 4 \times 96: 6]$ & $1 / 38.4$ & $20 / 3$ & 323.3 \\
$\chi_{\eta=1,2}[5 \times 56: 4 \times 112: 6]$ & $1 / 44.8$ & $40 / 7$ & 322.8 \\
$\chi_{\eta=1,2}[5 \times 64: 4 \times 128: 6]$ & $1 / 51.2$ & 5 & 322.5 \\
$\chi_{\eta=1,2}[5 \times 72: 4 \times 144: 6]$ & $1 / 57.6$ & $40 / 9$ & 322.2 \\
\hline \hline
\end{tabular}

TABLE II. Typical performance results for runs lasting 350M: number of processors, maximal memory requirement in GByte (to be precise, we quote the resident size of the program, i.e., the physical memory a task has used), total run time in CPU hours and average speed in $M / \mathrm{h}$ for the Altix 4700 of LRZ Munich [17] (using Intel Itanium2 Madison 9M CPUs running at $1.6 \mathrm{GHz})$.

\begin{tabular}{lccrr}
\hline \hline Grid configuration & Procs. & Mem. (GByte) & Time & $M / \mathrm{h}$ \\
\hline$\chi[5 \times 56: 4 \times 112: 6]$ & 10 & 8.9 & 192 & 18.2 \\
$\chi[5 \times 64: 4 \times 128: 6]$ & 12 & 11.8 & 306 & 13.7 \\
$\chi[5 \times 72: 4 \times 144: 6]$ & 14 & 17.5 & 505 & 9.7 \\
\hline \hline
\end{tabular}

frequency waves are concerned. To suppress highfrequency reflections at the mesh-refinement boundaries, which we have seen in quantities like $\Psi_{4}$ or the constraints, we use fourth-order Kreiss-Oliger dissipation as described in Sec. IV, where the factor $\sigma$ is chosen as $\sigma=0.1$ in the inner levels and $\sigma=0.5$ in the outer levels (where the waves are extracted).

\section{B. Results}

We have obtained fourth-order convergence for $r \Psi_{4}$ and the puncture tracks for sufficiently high resolution in the $\mathrm{BAM} \phi 1, \mathrm{BAM} \chi 1$, and $\mathrm{BAM} \chi 2$ series, requiring at least 48 grid points on the fine levels. For the $\operatorname{BAM} \phi 1$ series, low resolutions, e.g., with $\phi_{\eta=1}[4 \times i: 6 \times 2 i: 6], \quad i=$ $32,40,48$, show no systematic convergence behavior, while for the $\chi$ series, low resolutions show a convergence behavior as illustrated in the lower panel of Fig. 7: convergence is at least monotonic, but only the runs with high resolution are consistent with fourth-order convergence.

We now focus on the BAM $\chi 2$ series. The runs $\chi_{\eta=2}[5 \times$ $i: 4 \times 2 i: 6]$ show approximately second-order convergence for the set $i=32,40,48$, but achieve clean fourth-order convergence with $i=48,56,72$. The convergence results are shown in Figs. 7 and 8. Note that we see convergence in the waveforms without the time shift performed in [11]. In particular, the bottom plot in Fig. 7 shows the convergence of the time of the maximum amplitude in $r \Psi_{4}$ (extracted at $40 M$ ), as a function of the resolution on the finest box. We see that $t_{\max }=204.65 \pm 1.3 \mathrm{M}$ assuming 2nd-order convergence of the $i=32,40,48$ series, and $t_{\max }=203.9 \pm$ $0.2 M$ assuming 4th-order convergence for the $i=$ 48, 56, 72 series. These results are extremely accurate; compare, for example, with the results in [10,11].

A few comments about these results are in order. One might have stopped at the second-order convergent $i=$ 32, 40, 48 results indicated in the lower panel of Fig. 7 for the $\mathrm{BAM} \chi 2$ runs, since the code does have second-order ingredients (the initial-data calculation and interpolation in time at mesh-refinement boundaries). However, this theory is vetoed by finding that neither the accuracy of the initial data, nor the number of mesh-refinement buffer points, nor the size of the time step, have a significant influence on the 

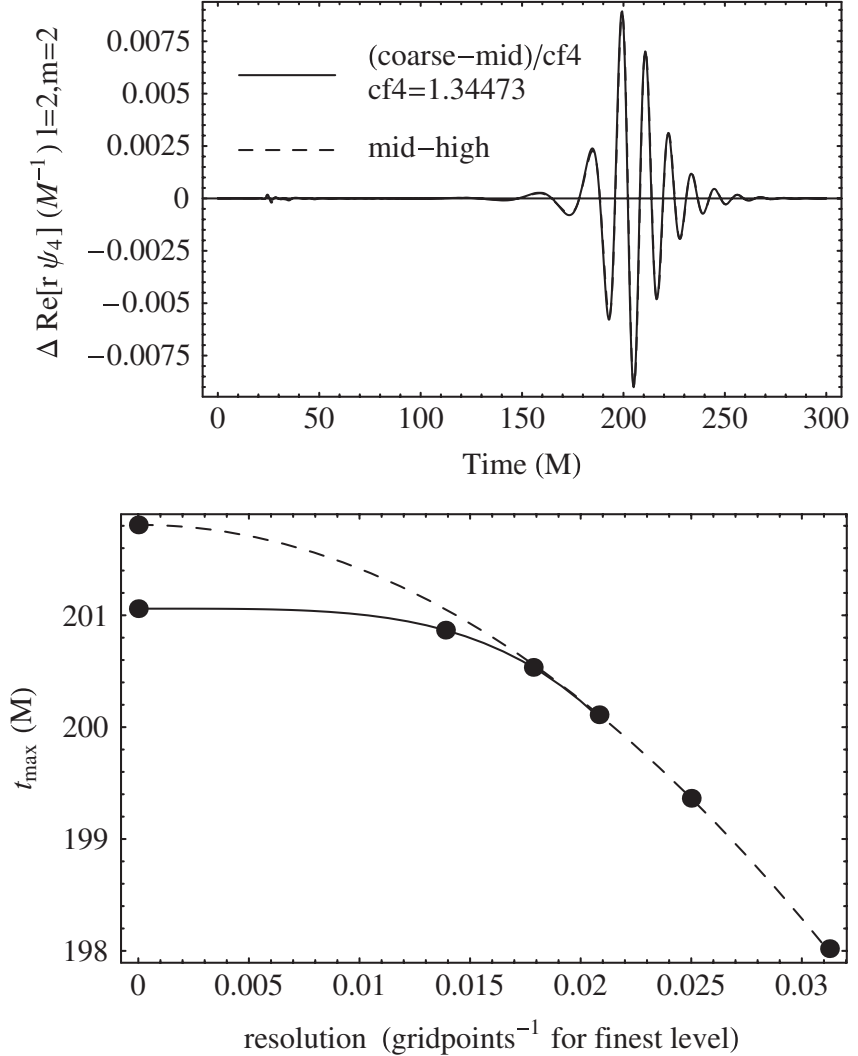

FIG. 7. Convergence of the $\operatorname{BAM} \chi_{\eta=2}[5 \times i: 4 \times 2 i: 6] \times$ $[i=32,40,48,56,72]$ series. Top: Scaled differences between results for different resolutions demonstrate fourth-order convergence. Bottom: The time $t_{\max }$ (in units of $M$ ) of the maximal amplitude in $\Psi_{4}$ is plotted versus resolution, and Richardsonextrapolated values are shown. The three most accurate runs $(i=$ 48,56 , and 72) show fourth-order convergence (solid line), while the runs with ( $i=32,40,48$, and 56) show second-order convergence (dashed line). The points on the far left are the Richardson-extrapolated values.

result. In such a situation it is necessary to increase the resolution in convergence tests, and indeed for higher resolutions fourth-order convergence was found. Note also that fourth-order convergence does not extend to the early (before approximately $t=125 \mathrm{M}$ ) and very late (after approximately $t=310 M$ ) parts of the waveform, where the errors are very small (Fig. 8), and to the late part of the puncture tracks after the merger as shown in Figs. 16 and 18-20. The late-time loss of convergence may be due to the location of the outer boundary, which is at $\sim 320 M$, and we expect incoming errors to reach the extraction radius (at $40 M$ ) after about $280 M$. It is encouraging that these errors are so small that they are detectable only in a convergence test, and that they do not have any effect on the stability of the runs.

Having established fourth-order convergence of our runs in the given regime, and having determined a grid and parameter setup that produces accurate results, we now perform an independent validation of our results by com-
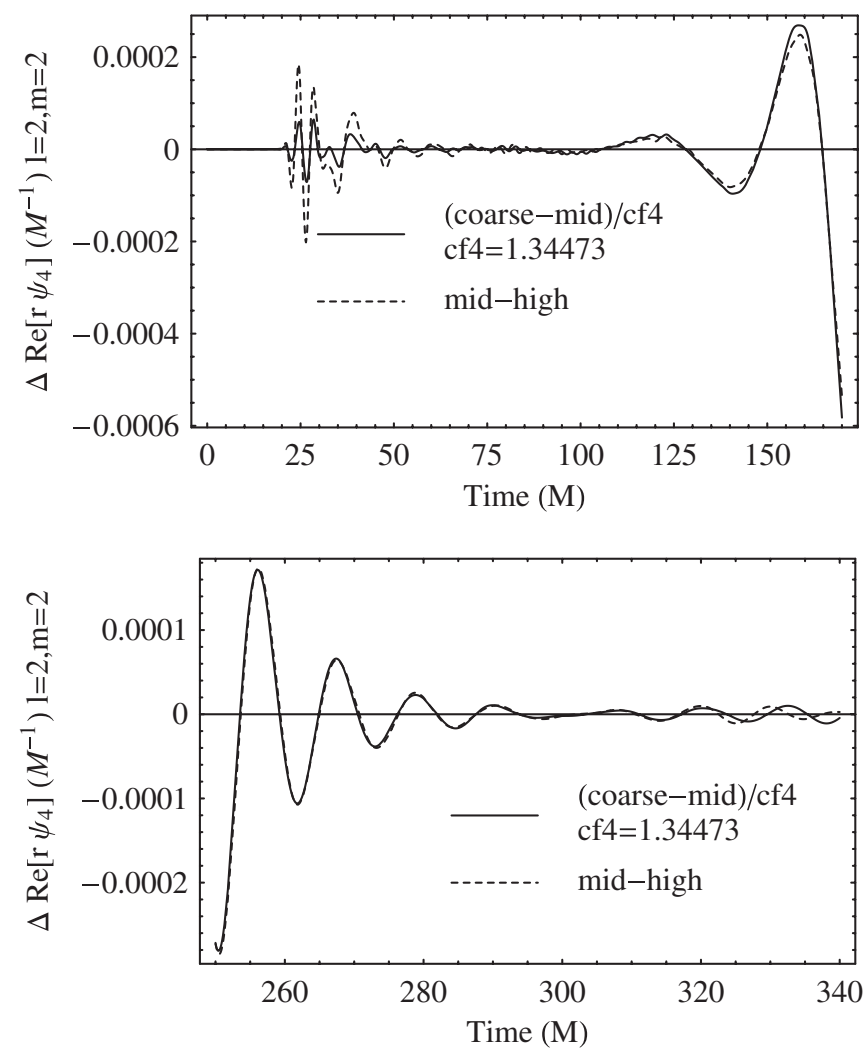

FIG. 8. Convergence of the $\operatorname{BAM} \chi_{\eta=2}[5 \times i: 4 \times 2 i: 6] \times$ $[i=48,56,72]$ series. Top: The early part of the waveform does not show fourth-order convergence until approximately $t=$ $125 \mathrm{M}$. Bottom: Clean fourth-order convergence is lost at approximately $t=310 \mathrm{M}$.

paring the $\Psi_{4}$ waveforms with those obtained with the LEAN code, as published in [13]. (The BAM and LEAN codes share a similar AMR grid structure, formulation of the Einstein equations, and numerical methods, and as such this is not a comparison of methods, only of codes; it is

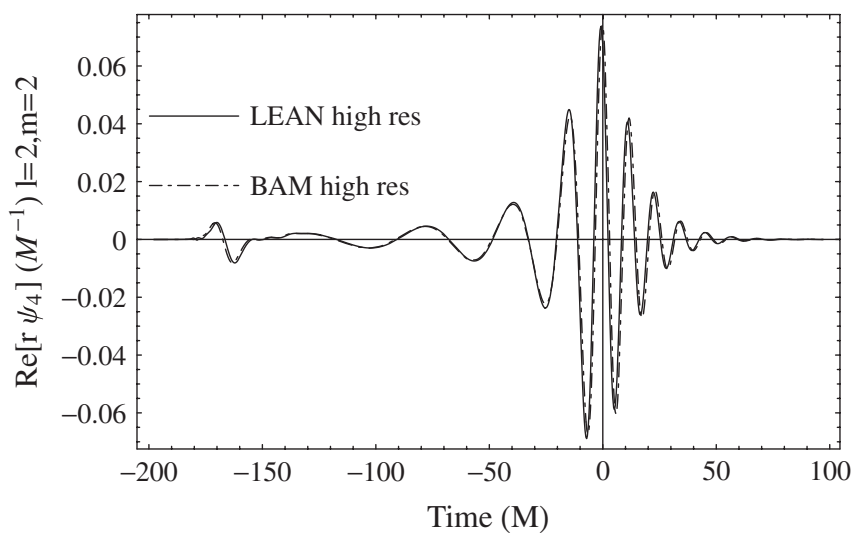

FIG. 9. Overlay of $\operatorname{Re}\left(r \Psi_{4}\right)$, extracted at $r=30$ for highest resolution LEAN $\phi_{\eta=1}$ (as published in [13]) and BAM $\chi_{\eta=2}[5 \times$ $72: 4 \times 144: 6][44.8,5.714]$ runs. The results have been shifted in time so $\max (|\Psi 4|)$ is aligned with $t=0$. 


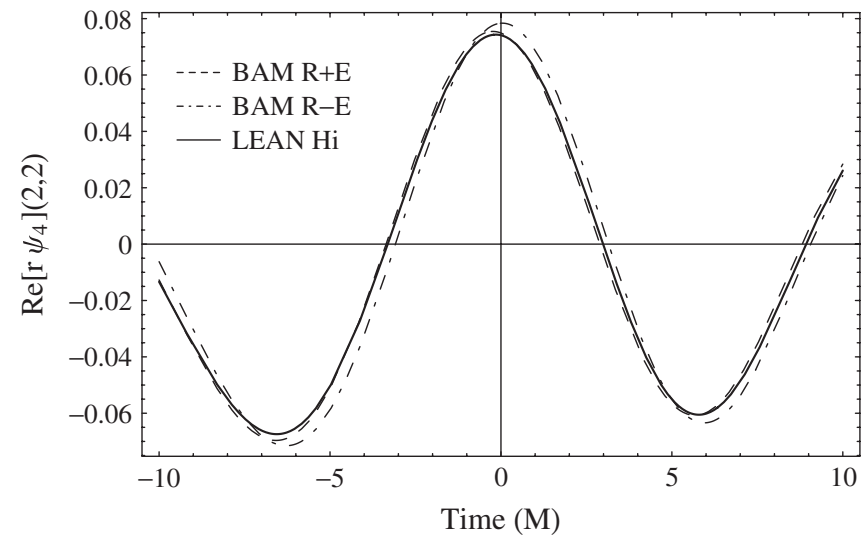

FIG. 10. The Richardson-extrapolated "main" part of the waveform is shown for the $\mathrm{BAM} \chi_{\eta=2}$ runs, error bars are obtained from the difference between Richardson-extrapolated result and the highest resolution run. The BAM result is overlaid with the highest resolution LEAN result. The results have been shifted in time so $\max (|\Psi 4|)$ is aligned with $t=0$.

meant to demonstrate that any remaining coding errors are negligible.) In the notation introduced above, the grid specifications of the high-resolution LEAN code run are $\phi_{\eta=1}[2 \times 72: 6 \times 130: 3][32.92: 3.47]$. This comparison is shown in Figs. 9 and 10. Figure 9 shows highest resolution BAM and LEAN $r \Psi_{4}, l=2, m=2$ modes, extracted at $r=$ 30 , for full runs. Figure 10 focuses on the main part of the waveform, and error bars are shown for the BAM results. We see that the results from the two codes show excellent agreement.

The main focus of this paper is to present and validate our code, and show that we are able to produce highly accurate results with moderate computational resources. In this spirit we present numerical error bars for our results, which are easily determined from the difference between highest resolution runs and Richardson-extrapolated values. However, waveforms and radiated energies also come with errors due to the finite extraction radius of the waves, and due to physically inappropriate boundary data. Figure 11 shows radiated energies versus time for $\chi_{\eta=2}$

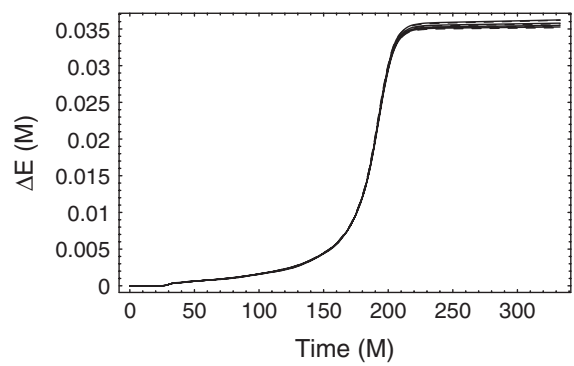

BAM runs $\chi_{\eta=2}[5 \times i: 4 \times 2 i: 6],(i=56,72)$ and extraction radii $r=25,30,35,40$. The dashed lines are from the $i=56$ run and the full lines from the $i=72$ run. The results have been shifted in time by the differences of extraction radii to minimize the phase difference. Clearly, the error from the variation of extraction radius is larger than numerical error at radii less than $30 \mathrm{M}$. Assuming that the error falls off with some power of $r$, a curve fit of our results suggests that the error falls off as $r^{2}$, and that in the $r \rightarrow \infty$ limit the extracted energy is $3.52 \%$. The value extracted at $r=40$ therefore has an error of only about $2 \%$, in contrast to the numerical error, which is less than $0.3 \%$. Further progress in accuracy obviously makes it very desirable to better model the falloff properties of the radiation. Although the only completely aesthetically pleasing solution would be to compactify at null infinity (see [73-79] for some recent work and overviews), one should expect that simple estimates based on perturbations of Kerr can be used to obtain significant improvements. A more detailed analysis goes beyond the scope of this paper, which focuses on the numerics, and will be published elsewhere.

The idea of extracting wave signals at finite distance from the source is that the timelike cylinder traced out by a sphere at sufficiently large distance from the source can be viewed as an approximation of null infinity, and increasing the distance will increase the quality of the approximation. Thus an approximation to the signal expected at a detector located at an astronomical distance from the source can be calculated. When the distance is of cosmological scale, cosmological redshift or further effects would have to be added "by hand." This idea raises several serious issues: the error introduced by the cutoff at finite radius needs to be estimated, "extrapolation procedures" to larger radius may yield significant improvements if a falloff law for the finite distance results can be assumed, and the gauge dependence of the results at finite distance needs to be addressed in order to optimize such procedures. It is therefore very valuable to compute results characterizing the asymptotics of the gravitational field by different methods,

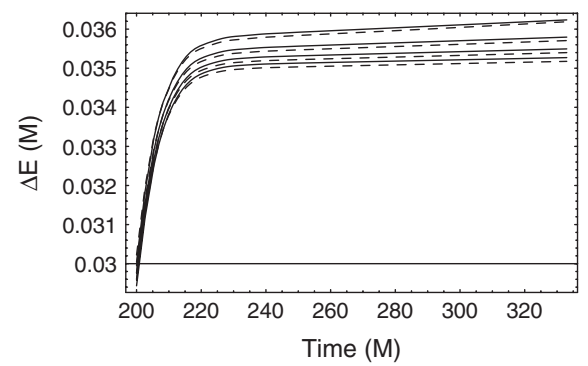

FIG. 11. Radiated energies plotted versus time for the runs $\chi_{\eta=2}[5 \times i: 4 \times 2 i: 6](i=56,72)$ and extraction radii $r=25$, 30, 35, 40. The dashed lines are from the $i=56$ run, the full lines from the $i=72$ run. Larger extraction radii yield smaller values for the radiated energy. The left image shows the result for the complete run, the right image zooms in on the late stage of the run. Results have been shifted in time by Eq. (61) to minimize the phase difference. Clearly, the error from the variation of extraction radius is not smaller than numerical error. 
which may show different effects from gauge, or different falloff laws, and compare the results of such different prescriptions of asymptotic quantities.

Along these lines, a good check on the consistency of the wave-extraction algorithm is to compare radiated energies with the energy balance that can be determined from evaluating the mass integral Eq. (55) at the beginning and end of the integration time. At finite extraction radius one can determine an estimate for the difference in Bondi mass; see Sec. III B. We have done this for all of our runs, and find excellent agreement for $\eta=1$, and less accurate results (approximately $4 \%$ of radiated energy, i.e., roughly $10 \%$ error) for $\eta=2$. The poor results for $\eta=2$ may be due to a drift in the coordinates. Such a drift was seen in the coordinate radius of the horizon in the Schwarzschild case in Sec. VA. For the case of orbiting punctures, we locate apparent horizons using a horizon finder based on the AHFINDER code in the CACTUS infrastructure [80]. Figures 12 and 13 show the motion of the apparent horizons for an orbital evolution (with punctures evolved from initial positions $y= \pm 3.5$ ), and also the coordinate radius of the single, and eventually common, apparent horizons as a function of time. We once again see an $\eta$-dependent coordinate drift, which we expect also affects the quality of the Bondi mass. However, note that such a strong gauge dependence is not seen for $\Psi_{4}$.

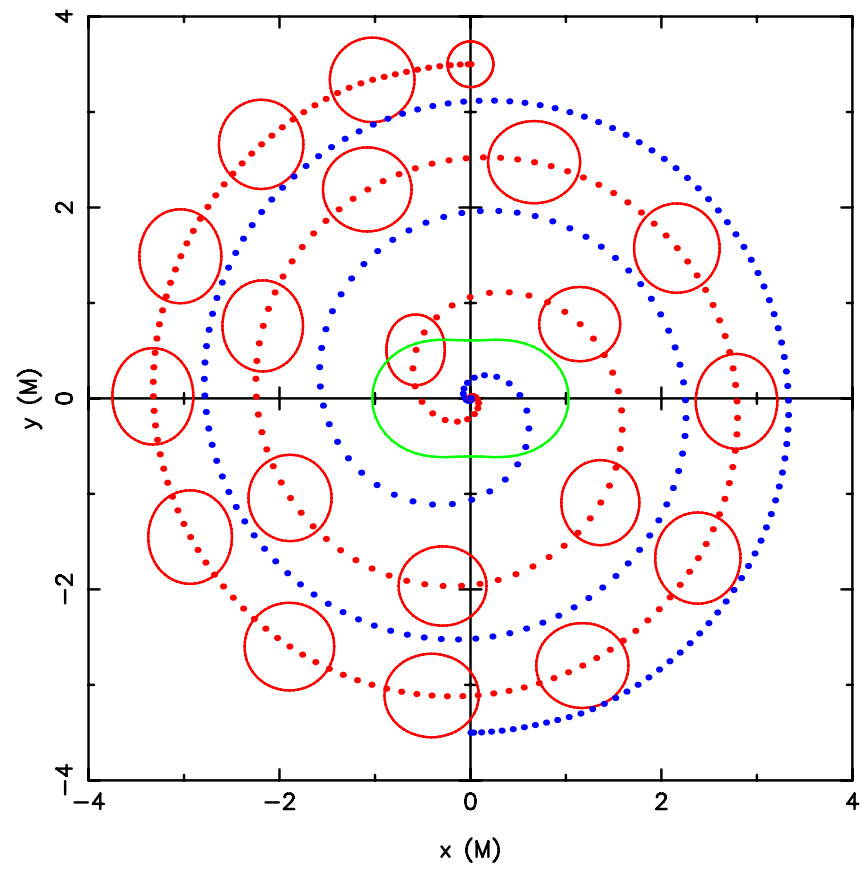

FIG. 12 (color online). Shape of the apparent horizon in the $x-y$ plane plotted each $10 M$ for a simulation with initial separation of $r=3.5 \mathrm{M}$. The dotted line represents the trajectory of the puncture. The behavior of the second horizon can be obtained by the symmetry of the problem. The common apparent horizon (peanut shaped in the figure) appears when the black holes have merged.

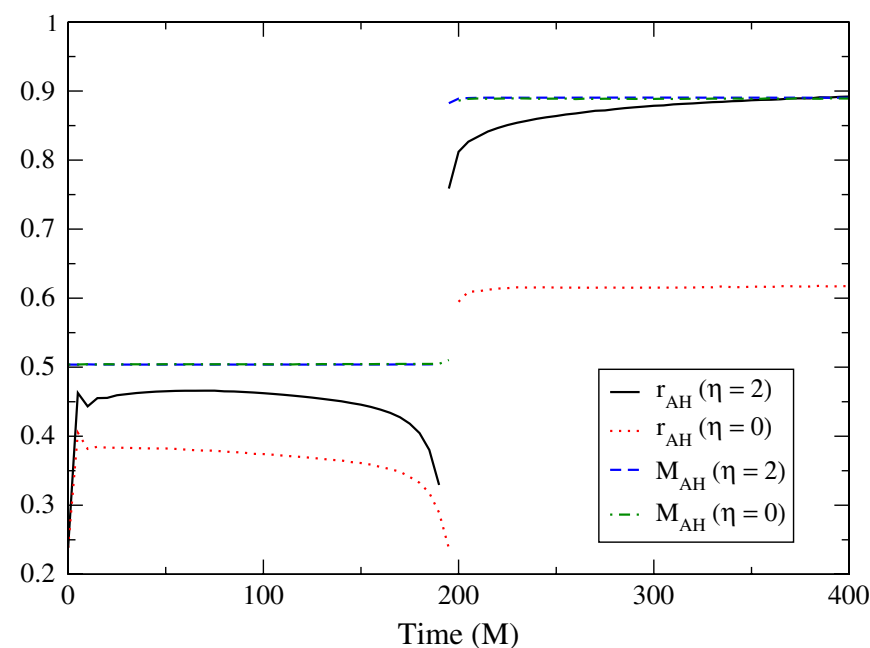

FIG. 13 (color online). Coordinate radius $r_{A H}$ of the apparent horizons as function of time for $\eta=0$ and $\eta=2$. The choice of $\eta$ has a strong influence in this gauge dependent quantity. The apparent-horizon mass $M_{A H}$ does not show any such gauge dependence - the respective curves are essentially on top of each other.

Important quantities to be determined are the amount of radiated angular momentum and the final spin of the black hole. For the time development of the angular momentum as determined from the surface integral Eq. (57); see Fig. 14. We determine the initial angular momentum by means of the surface integral Eq. (57), which can be evaluated analytically for Bowen-York data as $J_{0}=p D=$ 0.866 , corresponding to a Kerr parameter of $a / M_{0}=0.87$. We numerically calculate the final angular momentum as $J_{\text {final }}=0.634$, corresponding to a Kerr parameter of $a / M_{\text {final }}=0.688$ and radiated angular momentum of $25 \%$. The final angular momentum has been estimated by several methods with an error of roughly $1 \%$. The surface integral Eq. (57) gives results that are very accurate and

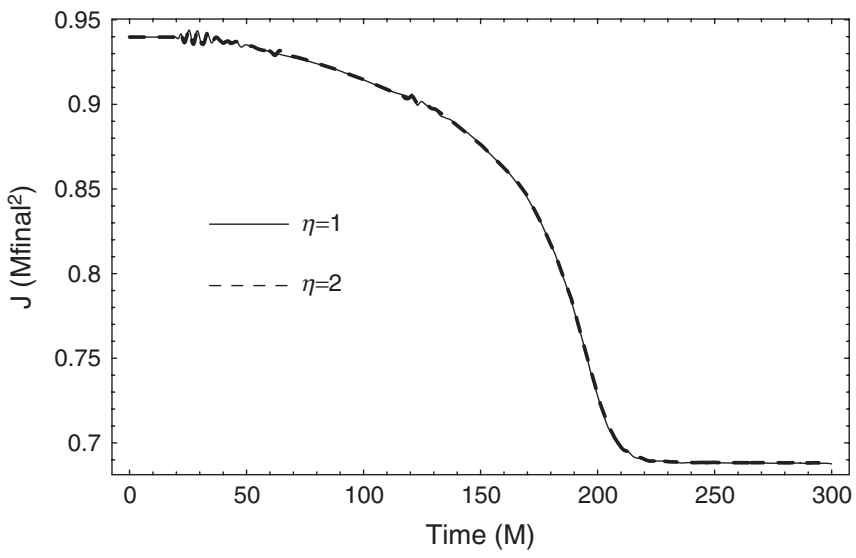

FIG. 14. Angular momentum computed at $r=30$ for gauge parameters $\eta=1,2$ with the $\mathrm{BAM}_{\chi}$ series - no significant dependency on the gauge is seen. 
consistent between different choices of the $\eta$ parameter. We also examine the complex quasinormal ring-down frequency. The real and imaginary part of the quasinormal ring-down frequency can be determined by directly fitting an exponentially damped sinusoid to the gravitational wave signal. The imaginary part can easily be read off from $\left|\Psi_{4}\right|$, which shows exponential falloff, and the real part of the frequency can be determined from the time derivative of the phase angle as defined in Eq. (54). In the limit of linearized theory, the real and imaginary parts of the frequency only depend on the mass and spin parameter of the final Kerr hole. This result, representing the "no-hair" theorem of general relativity, enables us to derive from the frequency values for the final mass and spin of the merged hole. In practice we use for that purpose tabulated values from Ref. [81].

All these results are consistent and yield a final spin of the black hole of $J=0.683 M_{\text {final }}^{2} \pm 1 \%$, where $M_{\text {final }}$ is the mass of the final black hole. Remarkably, the orbital frequency of the punctures levels off (see Fig. 15) to the real part of the quasinormal frequency at late times. Note that at early times, before the merger, the wave frequency has been observed to be twice the orbital frequency [11], as would naively be expected from the quadrupole formula.

One of the remarkable facts about recent simulations of binary black holes, whether done in a generalized harmonic or BSSN moving punctures framework, is the quality of the coordinate conditions: not only do they produce a "nice" spiraling motion with almost spherical (apart from a short time during merger) apparent horizons as seen in Fig. 12, but the coordinate tracks give rise to a good estimate of the waves via the quadrupole formula (see, for example, [82]), and the measured angular velocities coincide very accurately with what is expected on physical grounds. For example, at the beginning of the simulation the angular velocity quickly reaches a value close to that expected from the initial data (approximately $M \Omega=$ 0.05); see also [9]. A heuristic explanation for the latter fact has been given in [34]: symmetry-seeking gauge conditions (e.g., the gamma-freezing condition used here) should be expected to find an approximate helical Killing vector (HKV). This Killing vector will be unique up to a rigid rotation of the form $\vec{\omega} \times \vec{r}$. We can choose either corotation or vanishing rotation via the shift boundary condition at infinity. In this work the shift is set to zero at infinity. Thus the punctures' coordinate speeds are expected to be equal to their physical speeds seen from infinity. It is interesting to check whether the choice of gauge - in our approach this boils down to the choice of shift damping parameter $\eta$ - has an effect on the coordinate tracks of the punctures. We plot the radial and angular motion of the punctures with numerical errors (again determined from the difference of the Richardsonextrapolated value to highest resolution result) for the $\mathrm{BAM} \chi 2$ and $\mathrm{BAM} \phi 1$ runs in Fig. 15 . While the results
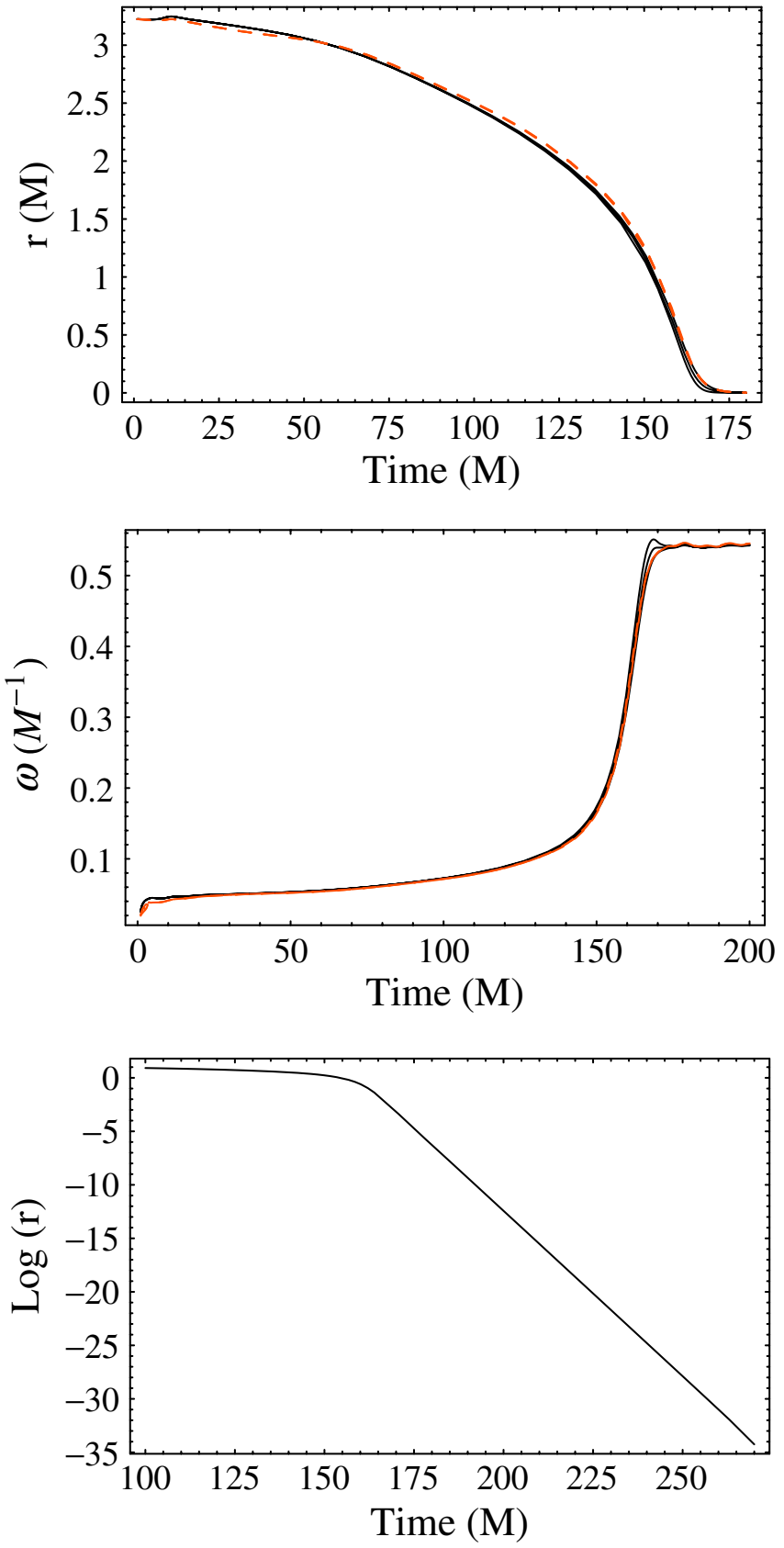

FIG. 15 (color online). Orbital motion with numerical errors obtained from difference of Richardson-extrapolated value to highest resolution result [top: $r(t)$; middle: $\omega(t)$ ]. The dashed curves represent $\chi_{\eta=2}$ and the solid curves $\phi_{\eta=1}$. The errors are so small that not all of the six lines in the plot are distinguishable; the main point is that the differences between the $\chi_{\eta=2}$ and $\phi_{\eta=1}$ results can be distinguished in $r(t)$, but not in $\omega(t)$. Bottom: The logarithm of the puncture radial position shows an exponential decay at late times, with a damping time of $\tau=$ $3.48 \pm 0.01\left(M_{\text {final }}\right)$.

look consistent between different runs, a small but significant $\eta$-dependent deviation can be seen in the radial motion, whereas a difference in angular motion is not visible in the plot. 
BERND BRÜGMANN et al.
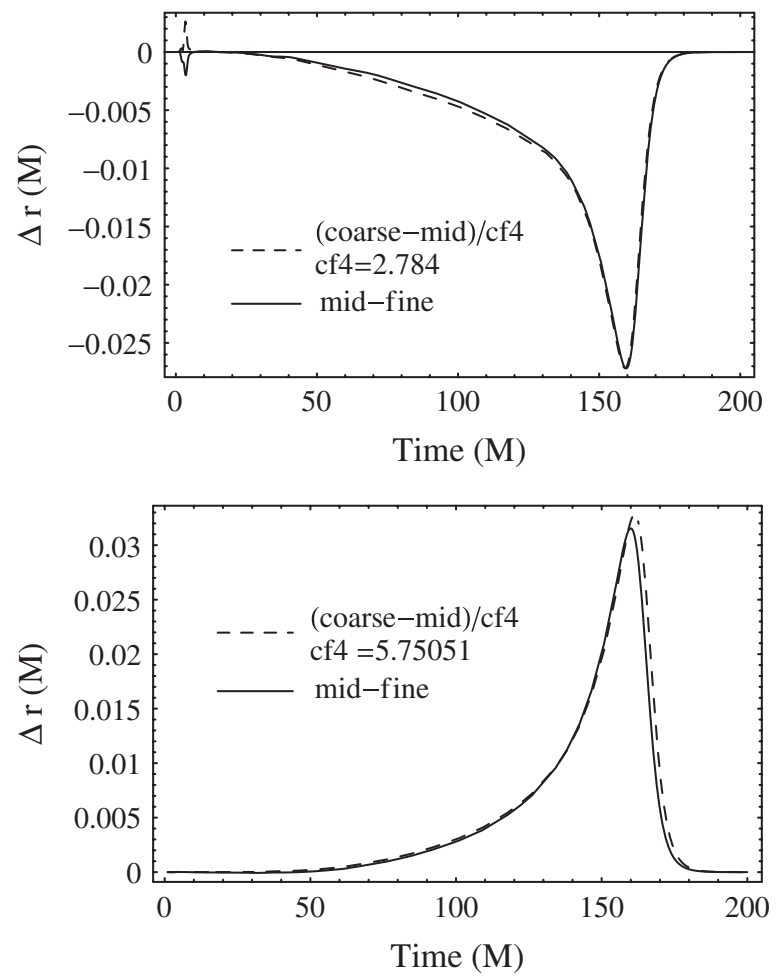

FIG. 16. Fourth-order convergence for $r(t)$ demonstrated for $\chi_{\eta=2}$ (top) and $\phi_{\eta=1}$ (bottom) series.
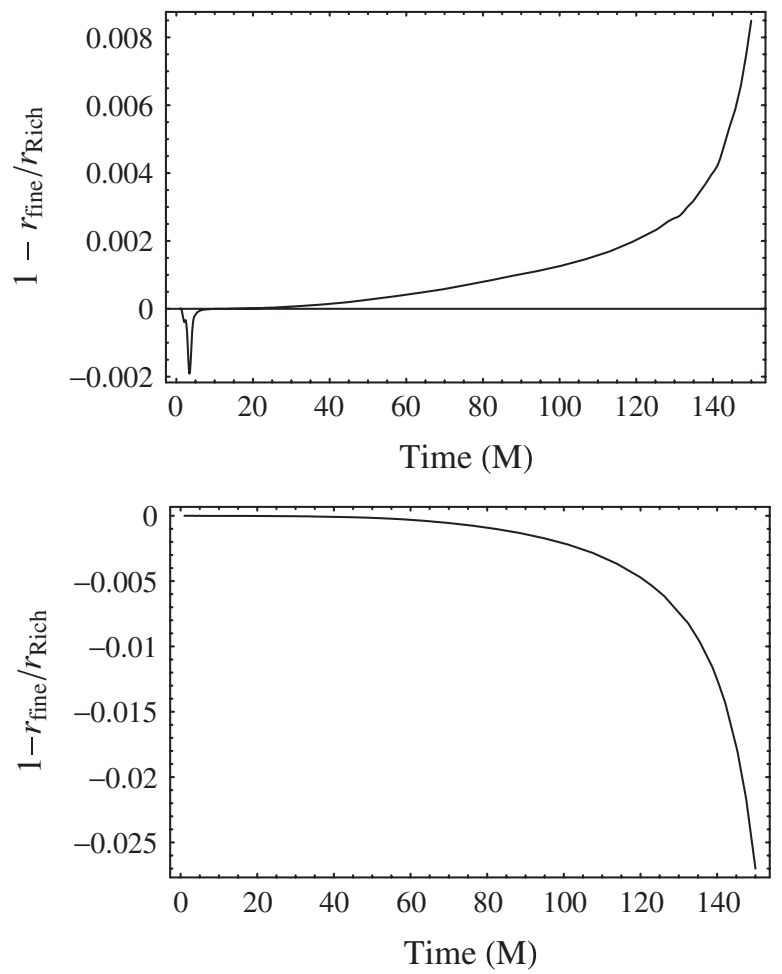

FIG. 17. Relative errors obtained from difference of Richardson-extrapolated value to highest resolution result (top: $\chi_{\eta=2}$; bottom: $\phi_{\eta=1}$ ).
PHYSICAL REVIEW D 77, 024027 (2008)
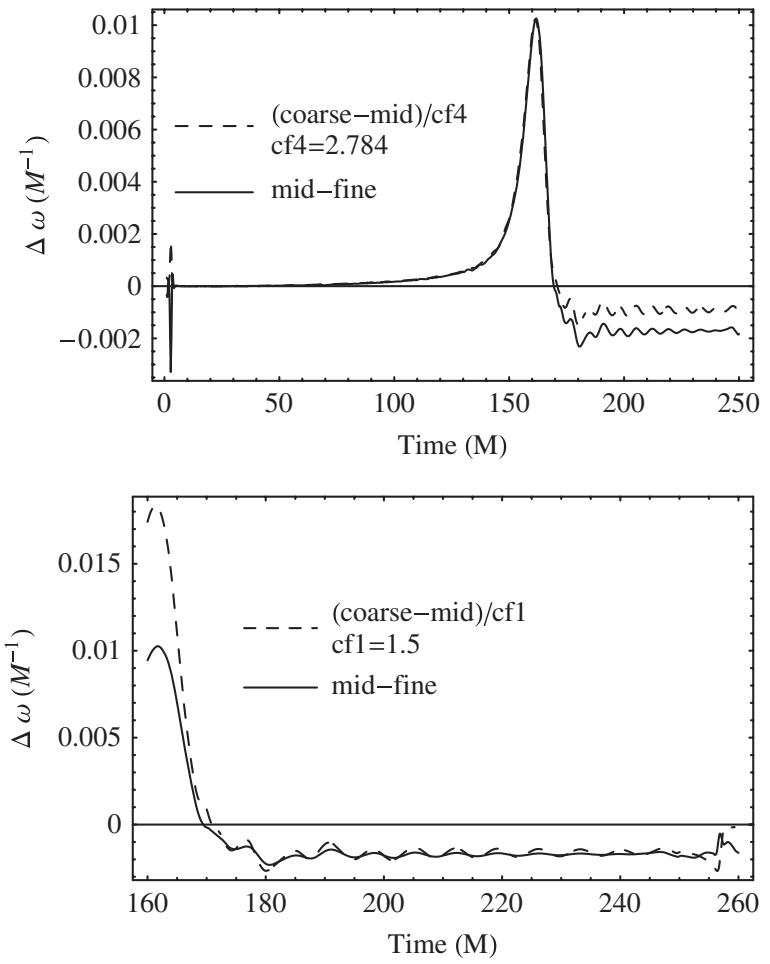

FIG. 18. Fourth-order convergence for $\omega(t)$ demonstrated for $\chi_{\eta=2}$ series, at late times only first-order convergence is seen.
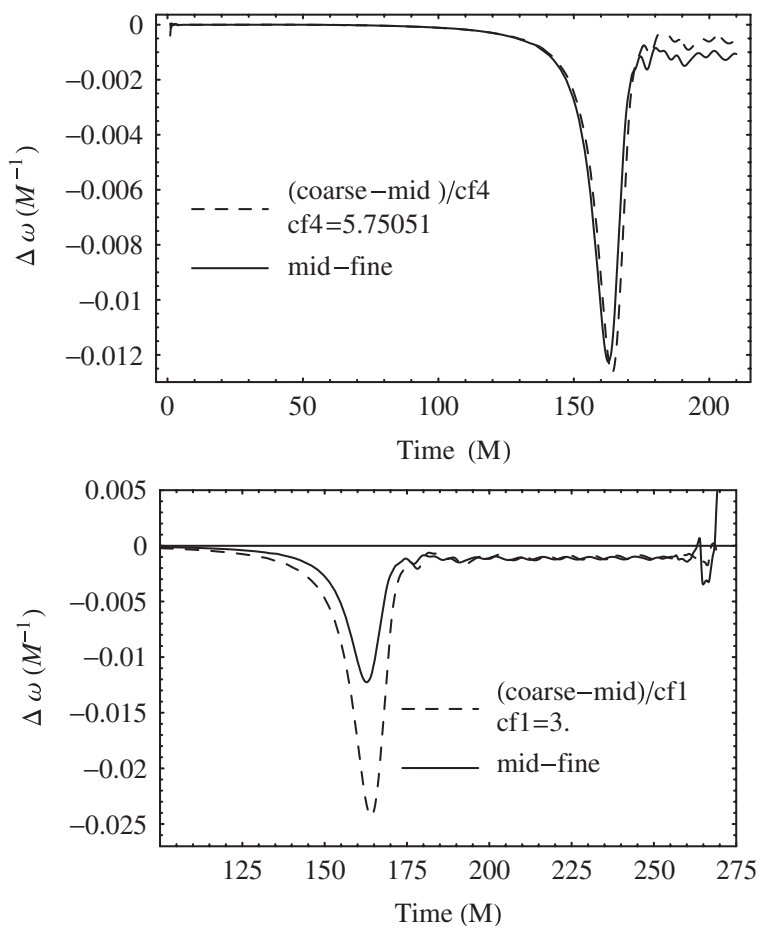

FIG. 19. Fourth-order convergence for $\omega(t)$ demonstrated for $\phi_{\eta=1}$ series, at late times only first-order convergence is seen. 

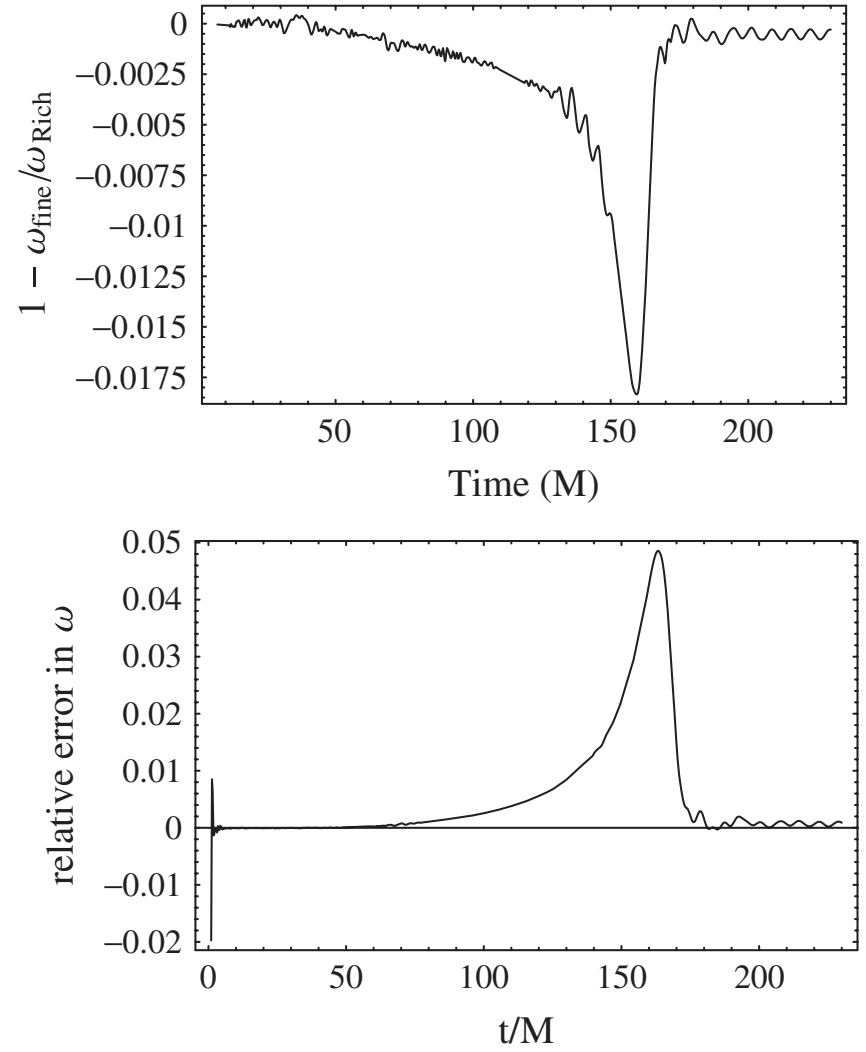

FIG. 20. Relative errors obtained from difference of Richardson-extrapolated value to highest resolution result (top: $\chi_{\eta=2}$; bottom: $\phi_{\eta=1}$ ).

Fourth-order convergence of the puncture motion in the $\chi 2$ and $\phi 1$ runs is demonstrated in Figs. 16-20. The coordinate angular speed $\omega$ is calculated using $\omega=$ $\left|\boldsymbol{\beta}_{i} \times \mathbf{n}\right| / r$, where $\mathbf{n}$ is the unit vector connecting the two punctures and $\boldsymbol{\beta}_{i}$ is the shift vector evaluated at the $i$ th puncture, which gives the coordinate speed of the puncture across the grid. This quantity shows fourth-order convergence up to the black-hole merger. After that time the puncture distance from the origin decays exponentially (see Fig. 15), thus rather soon the punctures are both less than one grid point from the origin and the convergence in $\omega$ deteriorates to first-order.

\section{QUASICIRCULAR ORBIT PARAMETERS}

For the runs in Sec. VI we chose the same initial parameters as used by Baker et al. [11] for their calibration runs. These are in turn based on those from Cook's 1994 initial-data study [83]. In that work the parameters for quasicircular orbits were determined using an "effectivepotential" (EP) method, whereby quasicircular orbits corresponding to a given total orbital angular momentum $J$ were identified by the minimum in a curve of the binding energy $E_{b}=E_{\mathrm{ADM}}-M_{1}-M_{2}$ versus the proper separation, in analogy with Newtonian physics. Cook's results applied to inversion-symmetric (not puncture) Bowen-
York data, but a later study of the innermost stable circular orbit of puncture Bowen-York data by Baumgarte [84] suggested that there are only minor (if any) physical differences between the two types of initial-data sets.

Note that Cook's parameters cannot be directly used for punctures. For this reason, the parameters we use come from Baker et al. [85] who have translated Cook's inversion-symmetric-data parameters for punctures. As pointed out in [86], this translation has introduced additional errors on the order of $1 \%$ in the masses, and the resulting data no longer precisely satisfy the quasicircular orbit requirements of the effective-potential method. This small error is not apparent in evolutions: the parameters used in [11] were found in practice to produce reasonably convincing quasicircular orbits. (At larger separations there is evidence of eccentricity, but this effect has also been observed for conformal thin-sandwich initial data [82].) In addition, Baker et al. [11] found that the merger waveform was largely independent of the initial separation of the punctures, suggesting that the translated Cook parameters really do correspond to points on an inspiral sequence.

However, there are a number of alternative ways to estimate the momenta as a function of separation for black holes on an inspiral sequence. One option is to use an approach based on the assumption of the existence of a HKV [86-88], for which a sequence of parameters for puncture data has been computed [28]. Another option is to use parameters predicted from PN theory, which were first used in the context of black-hole binary simulations in [85]. How sensitive is the final waveform to each of these approaches?

Let us first consider post-Newtonian parameters. For a given separation $D$, the momentum of each puncture can be given to 3PN order in the ADM-transverse-traceless (ADMTT) gauge by [89]

$$
\begin{aligned}
\frac{p}{\mu}= & \sqrt{\frac{M}{D}}+2 \epsilon\left(\frac{M}{D}\right)^{3 / 2}+\frac{1}{16} \epsilon^{2}(42-43 \nu)\left(\frac{M}{D}\right)^{5 / 2} \\
& +\frac{\epsilon^{3}}{128}\left[480+\left(163 \pi^{2}-4556\right) \nu+104 \nu^{2}\right]\left(\frac{M}{D}\right)^{7 / 2} .
\end{aligned}
$$

The total mass is $M=M_{1}+M_{2}$, the reduced mass is $\mu=$ $M_{1} M_{2} / M, \nu=\mu / M$, and the PN order of each term is indicated by $\epsilon$. For equal-mass black holes with $M_{1}=$ $M_{2}=0.5$, we have $\mu=\nu=0.25$.

Equation (65) was derived using Eqs. (5.1)-(5.3) in [89], and noting that $\omega_{\text {static }}=0$ and $\omega_{\text {kinetic }}=41 / 24$ [90]. Equations (5.1) and (5.3) can be rearranged, order by order in the post-Newtonian expansion, to give the orbital angular momentum $J$ as a function of puncture separation $D$, and we then use the relation $J=p D$ (which holds by definition; see Sec. IV of [89]) to write $p$ as a function of $D$. Equation (65) is not gauge invariant, but the ADMTT 
TABLE III. Initial parameters for a given initial coordinate separation, from three different approaches: the effectivepotential (EP) and helical Killing vector (HKV) methods, and the 3PN formula (65). The parameters are scaled with respect to the total black-hole mass in the initial data.

\begin{tabular}{lccc}
\hline \hline Method & $m / M$ & $y / M$ & $p / M$ \\
\hline EP & 0.4782 & 3.2248 & 0.1317 \\
HKV & 0.4782 & 3.2248 & 0.1307 \\
3PN & 0.4780 & 3.2248 & 0.1329 \\
\hline \hline
\end{tabular}

gauge is expected to be very close to the conformally flat gauge that we choose for our initial data.

Initial parameters from these three approaches, EP, HKV, and 3PN, are given in Table III. The parameters are scaled with respect to the total black-hole mass, $M=$ $M_{1}+M_{2}$, which is a convenient quantity in all three approaches; in the post-Newtonian expression (65) the black-hole masses appear, but the other standard mass scale, the total ADM mass $M_{\mathrm{ADM}}$, does not. In each case, the coordinate separation of the punctures is kept fixed, and a prediction for the momenta that will produce a quasicircular orbit is provided. These predictions all differ by less than $1 \%$ (which is also the error estimate in Cook's sequence [83]), and we might expect the resulting orbital motion and merger waveforms to be equally close. However, from Fig. 21 it is clear that this is not the case.

The black-hole merger times differ by about $40 M$, and the merger waveforms are noticeably different. However, from the tracks of the puncture locations during the evolutions, it is not clear which is the "better" choice of
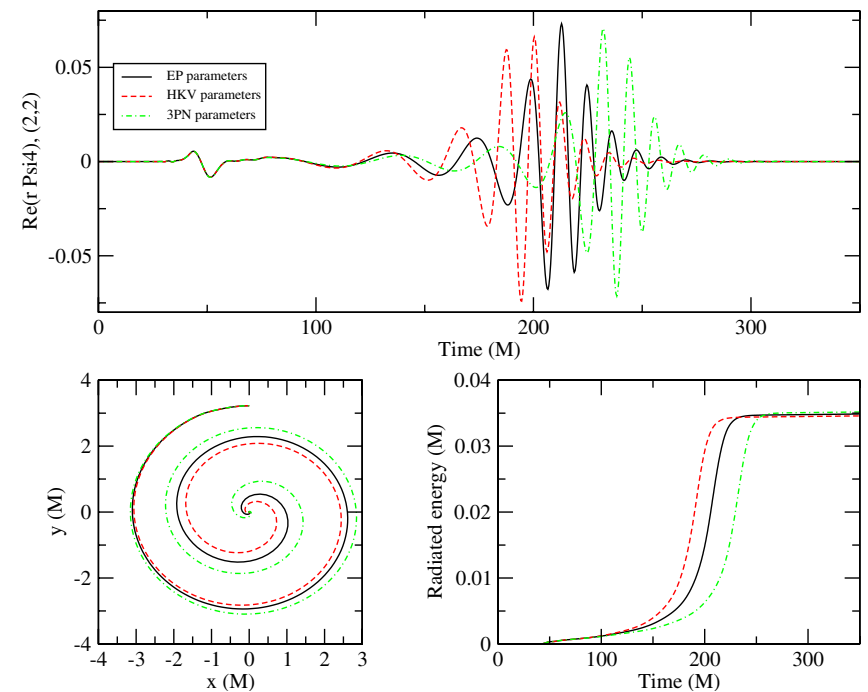

FIG. 21 (color online). Results from evolution of Bowen-York puncture data using three choices of initial parameters, described in the text. Top: Real part of the $l=2, m=2$ mode of $r \Psi_{4}$, extracted at $r=40 M$. Lower left: Paths followed by the punctures during evolution. Lower right: Energy extracted at $r=$ $40 M$ after $300 M$ of evolution.
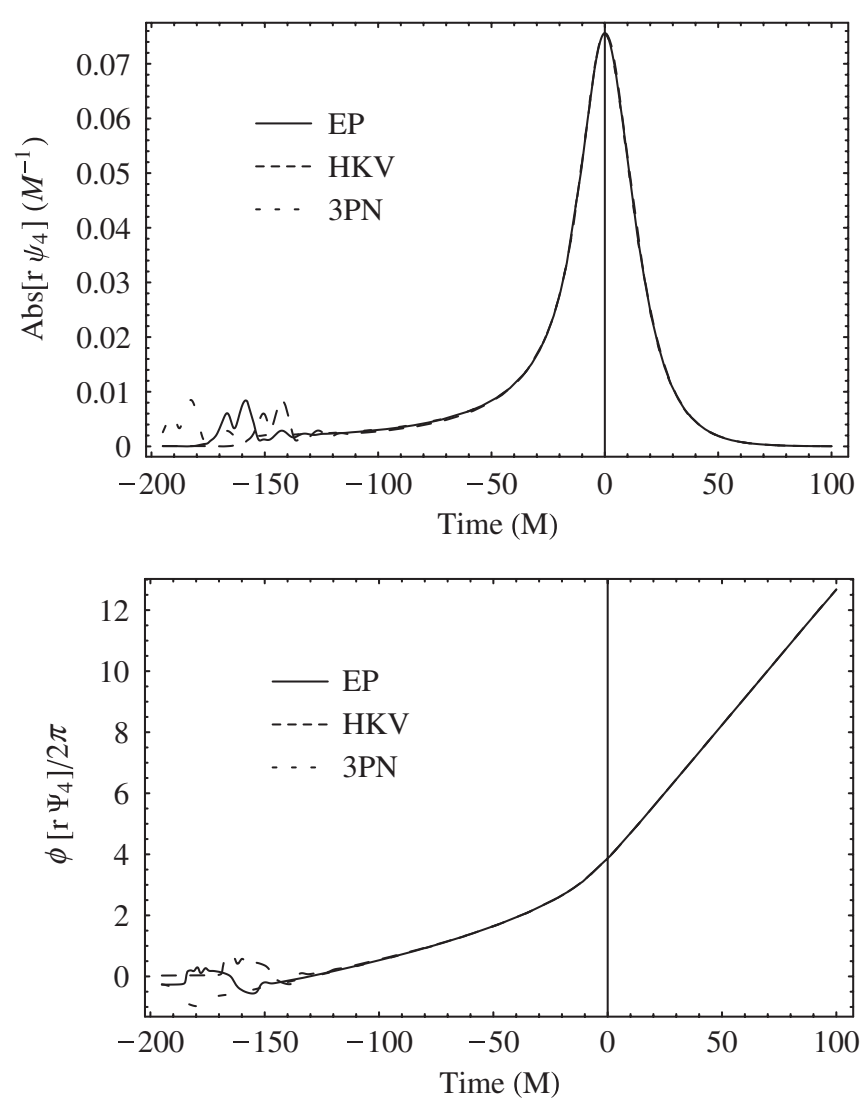

FIG. 22. Results from evolution of Bowen-York puncture data using three choices of initial parameters, described in the text. Top: Absolute value of the $l=2, m=2$ mode of $r \Psi_{4}$, extracted at $r=40 M$. Bottom: Phase angle (in units of $2 \pi$ ), shifted in time to align the maxima of $\operatorname{abs}\left(r \Psi_{4}\right)$ at $t=0$, and also aligning the phase at $t=0$.

quasicircular orbit parameters, or even what it would mean for one choice to be better than the other. These evolutions also suggest that parameters calculated from PN methods are an acceptable alternative to numerically generated parameters, allowing a wide range of configurations to be explored without the need for accompanying initialdata studies.

Despite the apparent differences in the dynamics, the radiated energy from the merger differs by only a few percent. In Fig. 22 the amplitude and phase of $\Psi_{4}$ are shown separately, with the results shifted in time so that the maximum in the amplitude occurs at the same time for all three choices of initial parameters (see $[11,85]$ ). It is now clear that, although the dynamics and waveforms look quite different, these differences are merely cosmetic: the physics of the merger is the same for all three choices of initial parameters.

\section{DISCUSSION}

We have presented a new code to evolve black-hole binaries, which is an extension of the older BAM code 
$[6,15,16]$. The new BAM code implements the "movingpuncture" method (in both its $\phi$ and $\chi$ versions) within a moving-box-based adaptive mesh-refinement grid structure, and uses fourth-order-accurate spatial finite differencing and RK4 time evolution. The primary analysis tool, the extraction of gravitational radiation waveforms, is implemented using the $\Psi_{4}$ Newman-Penrose scalar.

In this paper we have presented a number of important tests of our code: evolutions of a single nonspinning black hole, a single spinning black hole, and, the crucial test, evolutions of black-hole binaries. In addition to demonstrating fourth-order convergence in regimes of physical interest, each test has provided valuable insight into the grid sizes, resolutions, and geometries necessary to achieve accurate and efficient simulations. One of the ultimate uses of our code will be to perform large parameter studies of gravitational-wave sources, and therefore efficiency of the code is of equal concern to its accuracy and stability. The performance of the code, and how to tune its configuration to obtain good performance, is one of our main results: as shown in Table II we are able to perform a convergence series of three runs in the fourth-order convergent regime at a total cost of roughly $1000 \mathrm{CPU}$ hours and a maximal physical memory consumption of less than 18 GBytes. Accurate results for binary black-hole evolutions can thus already be obtained with relatively small commodity clusters, which are available to many research groups.

Evolutions of single black holes showed that both the $\phi$ and $\chi$ moving-puncture methods are stable and accurate, although the $\chi$ method shows better convergence properties at the puncture. The moving-puncture method was also found to be stable even when resolutions of up to $M / 512$ were used at the puncture. For black-hole binaries, the $\chi$ method showed monotonic convergence behavior (for example, in the merger times) at low resolutions, and appears to us as clearly preferable, even though we have not found prohibitive problems with the $\phi$ method. As a further element of validating our code, we have compared our waveform data with that from the independent LEAN code [13], and found that the two were in excellent agreement. This is a strong validation of both codes, and it will be interesting to see more extended comparisons between codes capable of performing binary black hole simula- tions - both regarding their results and efficiency in order to further refine the methods of the field.

We have investigated the influence of gauge choice, and have found consistent results between the 000 and $t t t$ shift advection choices, as well as the choice of the $\eta$ parameter in the $\tilde{\Gamma}$-driver shift condition, e.g., also by comparing the LEAN and BAM results. We have also found that, as expected, the $\eta$ parameter in the $\tilde{\Gamma}$-driver shift condition has an effect on the final coordinates of the solution, and as a result larger values of $\eta$ lead to a larger coordinate size of the black hole; in effect, the black hole becomes better resolved on the numerical grid. This coordinate drift may however also cause problems for naive wave extraction algorithms, and further research will be required to make optimal gauge choices.

Finally, we performed simulations using different choices of initial parameters for the momenta of the punctures. We found that very small changes in the initial momenta can make a large difference in the merger time of the black holes, but do not change the physical properties of the radiation.

Having carefully tested and calibrated our code for simulations of comparable-mass black-hole binaries, in future publications we plan to extend our research to parameter studies of unequal mass and spinning black holes, and to use initial-data sets with larger separations.

\section{ACKNOWLEDGMENTS}

It is a pleasure to thank Pedro Marronetti for discussions during the course of this project; Bernard Kelly for access to his unpublished notes on wave extraction; and Iris Christadler at the LRZ for assistance in code optimization. This work was supported in part by DFG Grant SFB/ Transregio 7 "Gravitational Wave Astronomy" and by NSF Grant No. PHY-0555644. S. H. acknowledges the hospitality of the relativity group at the University of the Balearic Islands, and has been supported in part by their CICYT Grant No. FPA-2004-03666. Computations where performed at HLRS, Stuttgart, and the LRZ, Munich. We also acknowledge partial support by the National Computational Science Alliance under Grant No. PHY060040T.
[1] S. G. Hahn and R. W. Lindquist, Ann. Phys. (N.Y.) 29, 304 (1964).

[2] L. Smarr, Ann. N.Y. Acad. Sci. 302, 569 (1977).

[3] F. Pretorius, Phys. Rev. Lett. 95, 121101 (2005).

[4] M. Campanelli, C. O. Lousto, P. Marronetti, and Y. Zlochower, Phys. Rev. Lett. 96, 111101 (2006).

[5] J. G. Baker, J. Centrella, D.-I. Choi, M. Koppitz, and J. van Meter, Phys. Rev. Lett. 96, 111102 (2006).
[6] B. Brügmann, W. Tichy, and N. Jansen, Phys. Rev. Lett. 92, 211101 (2004).

[7] M. A. Scheel, H. P. Pfeiffer, L. Lindblom, L. E. Kidder, O. Rinne, and S.A. Teukolsky, Phys. Rev. D 74, 104006 (2006).

[8] F. Pretorius, Classical Quantum Gravity 23, S529 (2006).

[9] P. Diener, F. Herrmann, D. Pollney, E. Schnetter, E. Seidel, R. Takahashi, J. Thornburg, and J. Ventrella, Phys. Rev. 
Lett. 96, 121101 (2006).

[10] M. Campanelli, C. O. Lousto, and Y. Zlochower, Phys. Rev. D 73, 061501(R) (2006).

[11] J. G. Baker, J. Centrella, D.-I. Choi, M. Koppitz, and J. van Meter, Phys. Rev. D 73, 104002 (2006).

[12] F. Herrmann, D. Shoemaker, and P. Laguna, arXiv:gr-qc/ 0601026.

[13] U. Sperhake, arXiv:gr-qc/0606079.

[14] S. Waldman, Classical Quantum Gravity 23, S653 (2006).

[15] B. Brügmann, Phys. Rev. D 54, 7361 (1996).

[16] B. Brügmann, Int. J. Mod. Phys. D 8, 85 (1999).

[17] HLRB2: SGI Altix 4700 system of LRZ Munich, http:// www.lrz-muenchen.de/services/compute/hlrb/.

[18] D. S. Brill and R.W. Lindquist, Phys. Rev. 131, 471 (1963).

[19] S. Dain, Lect. Notes Phys. 604, 161 (2002).

[20] J. W. York, in Sources of Gravitational Radiation, edited by L. L. Smarr (Cambridge University Press, Cambridge, England, 1979), pp. 83-126.

[21] H. P. Pfeiffer and J.W. York, Phys. Rev. D 67, 044022 (2003).

[22] J. M. Bowen and J. W. York, Phys. Rev. D 21, 2047 (1980).

[23] R. Beig and N. Ó Murchadha, Classical Quantum Gravity 11, 419 (1994).

[24] R. Beig and S. Husa, Phys. Rev. D 50, R7116 (1994).

[25] S. Brandt and B. Brügmann, Phys. Rev. Lett. 78, 3606 (1997).

[26] S. Husa, Ph.D. thesis, University of Vienna, 1998.

[27] S. Dain and H. Friedrich, Commun. Math. Phys. 222, 569 (2001).

[28] W. Tichy and B. Brügmann, Phys. Rev. D 69, 024006 (2004).

[29] D. Christodoulou, Phys. Rev. Lett. 25, 1596 (1970).

[30] M. Alcubierre, B. Brügmann, P. Diener, M. Koppitz, D. Pollney, E. Seidel, and R. Takahashi, Phys. Rev. D 67, 084023 (2003).

[31] M. Shibata and T. Nakamura, Phys. Rev. D 52, 5428 (1995).

[32] T. W. Baumgarte and S. L. Shapiro, Phys. Rev. D 59, 024007 (1998).

[33] M. Alcubierre, B. Brügmann, T. Dramlitsch, J. A. Font, P. Papadopoulos, E. Seidel, N. Stergioulas, and R. Takahashi, Phys. Rev. D 62, 044034 (2000).

[34] M. Hannam, S. Husa, D. Pollney, B. Brügmann, and N. Ó Murchadha, Phys. Rev. Lett. 99, 241102 (2007).

[35] C. Bona, J. Massó, E. Seidel, and J. Stela, Phys. Rev. D 56, 3405 (1997).

[36] J. R. van Meter, J. G. Baker, M. Koppitz, and D.-I. Choi, Phys. Rev. D 73, 124011 (2006).

[37] C. Gundlach and J. M. Martin-Garcia, Phys. Rev. D 74, 024016 (2006).

[38] L. T. Buchman and J.M. Bardeen, Phys. Rev. D 72, 124014 (2005).

[39] G. Nagy, O.E. Ortiz, and O.A. Reula, Phys. Rev. D 70, 044012 (2004).

[40] C. Gundlach and J. Martin-Garcia, Phys. Rev. D 70, 044031 (2004).

[41] C. Gundlach and J. M. Martin-Garcia, Phys. Rev. D 70, 044032 (2004).

[42] C. Gundlach and J. M. Martin-Garcia, Classical Quantum Gravity 23, S387 (2006).
[43] G. Calabrese, I. Hinder, and S. Husa, J. Comput. Phys. 218, 607 (2006).

[44] A. Nagar and L. Rezzolla, Classical Quantum Gravity 22, R167 (2005); http://stacks.iop.org/0264-9381/22/R167.

[45] C. W. Misner, K. S. Thorne, and J. A. Wheeler, Gravitation (W. H. Freeman, San Francisco, 1973).

[46] R. Gómez, L. Lehner, P. Papadopoulos, and J. Winicour, Classical Quantum Gravity 14, 977 (1997).

[47] W. Kinnersley, J. Math. Phys. (N.Y.) 10, 1195 (1969).

[48] D. R. Fiske, J. G. Baker, J. R. van Meter, D. Choi, and J. M. Centrella, Phys. Rev. D 71, 104036 (2005).

[49] J. Baker, M. Campanelli, and C. O. Lousto, Phys. Rev. D 65, 044001 (2002).

[50] Y. Wiaux, L. Jacques, and P. Vandergheynst, J. Comput. Phys. 226, 2359 (2007).

[51] Y. Zlochower, R. Gómez, S. Husa, L. Lehner, and J. Winicour, Phys. Rev. D 68, 084014 (2003).

[52] M. Campanelli and C. O. Lousto, Phys. Rev. D 59, 124022 (1999).

[53] R. Arnowitt, S. Deser, and C. W. Misner, in Gravitation: An Introduction to Current Research, edited by L. Witten (Wiley, New York, 1962), pp. 227-265.

[54] N. Ó Murchadha and J. W. York, Phys. Rev. D 10, 2345 (1974).

[55] J. W. York, Jr., Ann. Inst. Henri Poincaré, A 21, 319 (1974).

[56] H. Bondi, M. G. J. van der Burg, and A. W. K. Metzner, Proc. R. Soc. A 269, 21 (1962).

[57] J. I. Katz, D. Lynden-Bell, and W. Israel, Classical Quantum Gravity 5, 971 (1988).

[58] J. D. Brown, S. R. Lau, and J. W. York, Jr., Phys. Rev. D 55, 1977 (1997).

[59] E. Poisson, A Relativist's Toolkit: The Mathematics of Black-Hole Mechanics (Cambridge University Press, Cambridge, England, 2004).

[60] M. J. Berger and J. Oliger, J. Comput. Phys. 53, 484 (1984).

[61] Y. Zlochower, J. G. Baker, M. Campanelli, and C. O. Lousto, Phys. Rev. D 72, 024021 (2005).

[62] H.-O. Kreiss and J. Oliger, Methods for the Approximate Solution of Time Dependent Problems (World Meteorological Organization, International Council of Scientific Unions, 1973), Vol. 10.

[63] B. Gustafsson, H.-O. Kreiss, and J. Oliger, Time Dependent Problems and Difference Methods (Wiley, New York, 1995).

[64] E. Schnetter, S.H. Hawley, and I. Hawke, Classical Quantum Gravity 21, 1465 (2004).

[65] F. Pretorius and M. Choptuik, J. Comput. Phys. 218, 246 (2006).

[66] J.G. Baker and J.R. Meter, Phys. Rev. D 72, 104010 (2005).

[67] L. Lehner, S. L. Liebling, and O. Reula, Classical Quantum Gravity 23, S421 (2006).

[68] P. Csizmadia, Int. J. Mod. Phys. D 15, 107 (2006).

[69] J. W. York, Jr. and T. Piran, in Spacetime and Geometry: The Alfred Schild Lectures, edited by R. A. Matzner and L.C. Shepley (University of Texas Press, Austin, TX, 1982), pp. 147-176.

[70] M. W. Choptuik and W. G. Unruh, Gen. Relativ. Gravit. 18, 818 (1986). 
[71] S. Brandt and E. Seidel, Phys. Rev. D 54, 1403 (1996).

[72] B. Reimann and B. Brügmann, Phys. Rev. D 69, 044006 (2004).

[73] J. Winicour, Living Rev. Relativity 8, 10 (2005), http:// www.livingreviews.org/lrr-2005-10.

[74] S. Husa Lecture Notes in Physics (Springer, New York, 2002), Vol. 604, pp. 239-260.

[75] S. Husa, in The Conformal Structure of Spacetimes: Geometry, Analysis, Numerics, Lecture Notes in Physics Vol. 604, edited by J. Frauendiener and H. Friedrich (Springer-Verlag, Berlin, 2002).

[76] L. Andersson, Lect. Notes Phys. 604, 183 (2002).

[77] M. Babiuc, B. Szilagyi, I. Hawke, and Y. Zlochower, Classical Quantum Gravity 22, 5089 (2005).

[78] S. Husa, C. Schneemann, T. Vogel, and A. Zenginoglu, AIP Conf. Proc. 841, 306 (2006).

[79] C. Reisswig, N. T. Bishop, C. W. Lai, J. Thornburg, and B. Szilagyi, Classical Quantum Gravity 24, S327 (2007).

[80] M. Alcubierre, S. R. Brandt, B. Brügmann, C. Gundlach, J. Massó, E. Seidel, and P. Walker, Classical Quantum
Gravity 17, 2159 (2000).

[81] E. Berti, V. Cardoso, and C. M. Will, Phys. Rev. D 73, 064030 (2006).

[82] A. Buonanno, G. Cook, and F. Pretorius, Phys. Rev. D 75, 124018 (2007).

[83] G. B. Cook, Phys. Rev. D 50, 5025 (1994).

[84] T. W. Baumgarte, Phys. Rev. D 62, 024018 (2000).

[85] J. Baker, M. Campanelli, C. O. Lousto, and R. Takahashi, Phys. Rev. D 65, 124012 (2002).

[86] W. Tichy, B. Brügmann, and P. Laguna, Phys. Rev. D 68, 064008 (2003).

[87] E. Gourgoulhon, P. Grandclément, and S. Bonazzola, Phys. Rev. D 65, 044020 (2002).

[88] P. Grandclément, E. Gourgoulhon, and S. Bonazzola, Phys. Rev. D 65, 044021 (2002).

[89] T. Damour, P. Jaranowski, and G. Schäfer, Phys. Rev. D 62, 044024 (2000).

[90] T. Damour, P. Jaranowski, and G. Schäfer, Phys. Rev. D 62, 021501 (2000). 